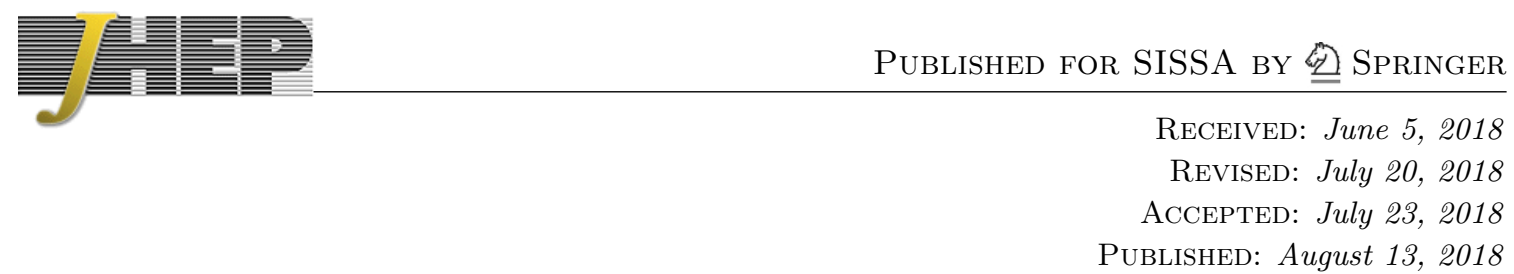

\title{
A realistic $U(2)$ model of flavor
}

\author{
Matthias Linster ${ }^{a}$ and Robert Ziegler ${ }^{a, b}$ \\ ${ }^{a}$ Institut für Theoretische Teilchenphysik, Karlsruhe Institute of Technology, \\ Engesserstraße 7, Karlsruhe, 76128 Germany \\ b Theoretical Physics Department, CERN, \\ Geneva 23, 1211 Switzerland \\ E-mail: matthias.linster@kit.edu, robert.ziegler@cern.ch
}

ABSTRACT: We propose a simple U(2) model of flavor compatible with an SU(5) GUT structure. All hierarchies in fermion masses and mixings arise from powers of two small parameters that control the $\mathrm{U}(2)$ breaking. In contrast to previous $\mathrm{U}(2)$ models this setup can be realized without supersymmetry and provides an excellent fit to all SM flavor observables including neutrinos. We also consider a variant of this model based on a $D_{6} \times \mathrm{U}(1)_{F}$ flavor symmetry, which closely resembles the $\mathrm{U}(2)$ structure, but allows for Majorana neutrino masses from the Weinberg operator. Remarkably, in this case one naturally obtains large mixing angles in the lepton sector from small mixing angles in the quark sector. The model also offers a natural option for addressing the Strong CP Problem and Dark Matter by identifying the Goldstone boson of the $\mathrm{U}(1)_{F}$ factor as the QCD axion.

KeYwords: Quark Masses and SM Parameters, Neutrino Physics

ARXiv EPrint: 1805.07341

Open ACCEss, (c) The Authors.

Article funded by $\mathrm{SCOAP}^{3}$.

https://doi.org/10.1007/JHEP08(2018)058 


\section{Contents}

1 Introduction 1

2 A realistic $U(2)$ model of flavor 3

2.1 Quark and charged lepton sector 3

$\begin{array}{lll}2.2 & \text { Fit to quark and charged lepton sector } & 6\end{array}$

2.3 Neutrino sector 8

3 A $D_{6} \times \mathrm{U}(1)$ model of flavor $\quad 13$

$\begin{array}{lll}3.1 \text { Setup } & 13\end{array}$

$\begin{array}{ll}3.2 & \text { Numerical fit } \\ \end{array}$

4 The U(2) axiflavon $\quad 17$

$\begin{array}{lll}4.1 \text { Axion couplings } & 18\end{array}$

$\begin{array}{lll}4.2 & \text { Axion phenomenology } & 20\end{array}$

5 Summary and conclusions $\quad 22$

$\begin{array}{ll}\text { A } D_{3} \text { and } D_{6} \text { group theory } & 25\end{array}$

$\begin{array}{ll}\text { B Fit results } & 27\end{array}$

$\begin{array}{lr}\text { C Scalar potential } & 27\end{array}$

\section{Introduction}

One of the prominent problems of the Standard Model (SM) is the presence of large hierarchies in fermion masses and mixings. Even neglecting neutrino masses, which might have a different origin, the Yukawa couplings span a range from $10^{-6}$ for the electron up to unity for the top quark. Mixing angles in the quark sector are small and hierarchical, while all mixing angles in the lepton sector are sizable. Explaining these hierarchies is referred to as the "SM Flavor Puzzle" (see e.g. ref. [1] for a review).

A popular framework to address this problem is in terms of approximate flavor (or horizontal) symmetries. The SM fermions are charged under this symmetry, so that most of the Yukawa couplings are forbidden in the symmetry limit. The flavor symmetry is spontaneously broken by vacuum expectation values of scalar fields (the so-called flavons), which allows to estimate the Yukawa couplings using a spurion analysis. Within an effective field theory approach, appropriate powers of flavon insertions are needed to make a given Yukawa operator invariant under the flavor symmetry, suppressed by some large UV cutoff scale. The flavon VEVs are assumed to be slightly below this cutoff scale, so that SM 
Yukawas arise from powers of these small order parameters. The effective operators have coefficients that are not predicted by the model, but should not be too large or small, in order to explain all hierarchies with the approximate flavor symmetry alone.

While a plethora of this kind of models have been constructed (see e.g. ref. [1] and references therein), a particularly simple and interesting class of models is based on a $\mathrm{U}(2)$ flavor symmetry $[2,3]$. In the original model the flavor quantum numbers are compatible with an SO(10) GUT structure, and therefore viable only in a supersymmetric (SUSY) context (or more generally in models with at least one additional Higgs field, needed to account for the $m_{b} / m_{t}$ hierarchy). Holomorphy together with the $\mathrm{U}(2)$ breaking pattern by two spurions then leads to three texture zeros in the quark mass matrices, which imply certain relations between CKM mixing angles and quark masses, in particular $V_{u b} / V_{c b}=$ $\sqrt{m_{u} / m_{c}}$. Unfortunately, this prediction is incompatible with the current experimental precision of $V_{u b}$ and $V_{c b}$, and this simple and economic model was ruled out [4] with the advent of $B$-factories.

Therefore modifications of the original model have been proposed in order to modify the model predictions and comply with experimental data. In ref. [5] a SUSY SO(10) model with a $D_{3} \times \mathrm{U}(1)$ flavor symmetry was studied, which mimicked the original $\mathrm{U}(2)$ structure with three texture zeros, but is also in conflict with present values of CKM elements. A more recent study has been performed in ref. [6], which has shown that the problematic relation can be fixed by taking flavor quantum numbers compatible only with an SU(5) GUT structure. This allows the presence of large rotations in the right-handed (RH) down sector that correct the predictions, as suggested in ref. [4]. Relaxing the $\mathrm{SO}(10)$ structure admits to consider also non-supersymmetric models, and in ref. [7] such a model was constructed with a charged lepton sector designed to address the (still existing) anomalies in semileptonic $B$-meson decays. This requires to give up also the $\mathrm{SU}(5)$ compatibility, but the model can successfully explain the observed deviations in $R_{K}$ [8] and $R_{K^{*}}$ [9] by the tree-level exchange of a $Z^{\prime}$ boson in the TeV range. In contrast to many $Z^{\prime}$ models that address the anomalies, the couplings to fermions are related to the flavor sector and thus essentially predicted in terms of fermion masses and mixings.

In this work we build upon the previous studies in refs. [2,6,7] and propose a simple, non-supersymmetric $\mathrm{U}(2)$ model of flavor that is compatible with an SU(5) GUT structure. The problematic relations between CKM mixing angles and quark masses are modified due to large mixing angles in the RH down sector, allowing for an excellent fit to CKM angles and quark and charged lepton masses. All hierarchies arise from powers of two small parameters (roughly of the same order) describing the $\mathrm{U}(2)$ breaking pattern. We also include the neutrino sector, which in this framework can be straightforwardly reproduced by adding three light SM singlets with suitable $\mathrm{U}(2)$ quantum numbers and Dirac masses. The fit to the full SM fermion sector is excellent, and predicts the overall mass scale in the neutrino sector below current cosmological bounds. We further discuss a variant of the $\mathrm{U}(2)$ model where the $\mathrm{SU}(2)$ factor is replaced by the discrete group $D_{6}$. The breaking pattern and the resulting Yukawa matrices closely resemble the $\mathrm{SU}(2)$ case. The only difference is a flipped sign in the 1-2 entry of the mass matrices, which has no effect in the quark and charged lepton sector, but allows to obtain Majorana neutrinos masses from the 
Weinberg operator. In contrast to the Dirac case the parametric flavor suppression of the neutrino mass matrix is fixed purely by charged lepton charges. Remarkably, this matrix is automatically anarchical, and therefore allows for an excellent fit to neutrino data, again predicting the overall neutrino mass scale in about the same range as in the Dirac case.

Finally we discuss the fate of the $\mathrm{U}(1) \subset \mathrm{U}(2)$ Goldstone boson, which naturally plays the role of the QCD axion and has (flavor-violating) couplings to fermions that are predicted by the flavor model, in the spirit of refs. [10-12]. In contrast to single U(1) flavor models, here the additional $\mathrm{SU}(2)$ flavor symmetry protects flavor-violating couplings to light generations (much as in SUSY U(2) models $[6,7]$ ), so that the resulting axion is mainly constrained by astrophysics and not by precision flavor observables. It is well-known that the axion can be an excellent Dark Matter (DM) candidate for large ranges of the $\mathrm{U}(1)$ breaking scale, which here is directly connected to the UV cutoff of the flavor model. In this way the model offers a natural solution for the strong CP problem and the origin of DM.

This paper is organized as follows. In section 2 we define the $\mathrm{U}(2)$ flavor model and discuss the structure of the quark and charged lepton sector before addressing the (Dirac) neutrino sector. We then consider a $D_{6} \times \mathrm{U}(1)$ model in section 3 , which closely follows the $\mathrm{U}(2)$ structure and allows to obtain Majorana neutrino masses from the Weinberg operator. In section 4 we address the Strong CP Problem and Dark Matter within this framework, interpreting the Goldstone boson of the U(1) factor as the QCD axion. We finally conclude in section 5 . In three appendices we provide more details on the group theoretical structure of $D_{3}$ and $D_{6} \simeq D_{3} \times Z_{2}$, include more details about the numerical fit, and discuss an explicit example of the scalar potential generating the flavon VEVs in the $D_{6} \times \mathrm{U}(1)$ model.

\section{A realistic $\mathrm{U}(2)$ model of flavor}

In this section we define our framework and show how hierarchies in the quark and charged lepton sector arise from the $\mathrm{U}(2)$ flavor symmetry. After discussing the analytical relations between CKM elements and quarks masses, we perform a numerical fit to masses and mixings. We then address the neutrino sector in the context of Dirac neutrinos and include it in the numerical fit. We conclude this section with a general discussion of the flavor structure of neutrino masses, motivating the $D_{6} \times \mathrm{U}(1)$ flavor model in the next section.

\subsection{Quark and charged lepton sector}

We consider an extension of the SM with a global flavor symmetry group $\mathrm{U}(2)_{F}$. Locally this group is isomorphic to $\mathrm{SU}(2)_{F} \times \mathrm{U}(1)_{F}$, under which $\mathrm{SM}$ fermions are charged. This symmetry group is assumed to be broken slightly below a UV scale $\Lambda$, which sets the relevant mass scale for additional dynamics. We also assume that the scale $\Lambda$ is large enough to safely neglect the impact of these new degrees of freedom on phenomenology. Thus, we simply work with an effective theory with cut-off scale $\Lambda$ that only involves SM fields and spurions that parametrize the breaking of $\mathrm{SU}(2)_{F} \times \mathrm{U}(1)_{F}$. 


\begin{tabular}{|cccccccc|}
\hline & $\mathbf{1 0}_{a}$ & $\overline{\mathbf{5}}_{a}$ & $\mathbf{1 0}_{3}$ & $\overline{\mathbf{5}}_{3}$ & $H$ & $\phi_{a}$ & $\chi$ \\
\hline $\mathrm{SU}(2)_{F}$ & $\mathbf{2}$ & $\mathbf{2}$ & $\mathbf{1}$ & $\mathbf{1}$ & $\mathbf{1}$ & $\mathbf{2}$ & $\mathbf{1}$ \\
$\mathrm{U}(1)_{F}$ & 1 & 1 & 0 & 1 & 0 & -1 & -1 \\
\hline
\end{tabular}

Table 1. The field content and $\mathrm{U}(2)_{F}$ quantum numbers.

The SM fermions have $\mathrm{U}(2)_{F}$ quantum numbers that are compatible with an $\mathrm{SU}(5)$ GUT structure, i.e. they are specified by the quantum number of the two SU(5) representations $\mathbf{1 0}=Q, U, E$ and $\overline{\mathbf{5}}=L, E$. The first two generations transform as a doublet under $\mathrm{SU}(2)_{F}$, the third generation is an $\mathrm{SU}(2)_{F}$ singlet and the Higgs field is a singlet under both $\mathrm{SU}(2)_{F}$ and $\mathrm{U}(1)_{F}$. Thus, the $\mathrm{U}(1)_{F}$ quantum numbers of the SM fermions are specified by four charges $\left\{X_{10_{a}}, X_{\overline{5}_{a}}, X_{10_{3}}, X_{\overline{5}_{3}}\right\}$ for $\left\{\mathbf{1 0}_{a}, \overline{\mathbf{5}}_{a}, \mathbf{1 0}_{3}, \overline{\mathbf{5}}_{3}\right\}$ with $a=1$, 2. It turns out that a successful fit to the observed fermion masses and mixings can be achieved for the following simple choice for $\mathrm{U}(1)_{F}$ charges:

$$
X_{10_{3}}=0, \quad X_{10_{a}}=X_{\overline{5}_{a}}=X_{\overline{5}_{3}}=1 .
$$

The breaking of the flavor symmetry is described by two scalar spurions $\phi$ and $\chi$, which transform under $\mathrm{U}(2)_{F}$ as $\phi=\mathbf{2}_{-1}$ and $\chi=\mathbf{1}_{-1}$. These fields acquire the following vacuum expectation values (VEVs):

$$
\langle\phi\rangle=\left(\begin{array}{c}
\varepsilon_{\phi} \Lambda \\
0
\end{array}\right), \quad\langle\chi\rangle=\varepsilon_{\chi} \Lambda
$$

where we will take $\varepsilon_{\phi} \sim \varepsilon_{\chi} \sim \mathcal{O}(0.01)$. In table 1 we summarize the field content and the transformation properties under the flavor group. As the fermions are charged under $\mathrm{U}(2)_{F}$, Yukawa couplings require additional spurion insertions in order to be $\mathrm{U}(2)_{F}$-invariant. This leads to non-renormalizable interactions suppressed by appropriate powers of $\Lambda$. For example, the resulting Lagrangian in the up-sector, at leading order in $\varepsilon_{\phi, \chi}$, is given by

$$
\begin{aligned}
\mathcal{L}_{u}= & \frac{\lambda_{11}^{u}}{\Lambda^{6}} \chi^{4}\left(\phi_{a}^{*} Q_{a}\right)\left(\phi_{b}^{*} U_{b}\right) H+\frac{\lambda_{12}^{u}}{\Lambda^{2}} \chi^{2} \epsilon_{a b} Q_{a} U_{b} H+\frac{\lambda_{13}^{u}}{\Lambda^{3}} \chi^{2}\left(\phi_{a}^{*} Q_{a}\right) U_{3} H \\
& +\frac{\lambda_{22}^{u}}{\Lambda^{2}}\left(\epsilon_{a b} \phi_{a} Q_{b}\right)\left(\epsilon_{c d} \phi_{c} U_{d}\right) H+\frac{\lambda_{23}^{u}}{\Lambda}\left(\epsilon_{a b} \phi_{a} Q_{b}\right) U_{3} H+\frac{\lambda_{31}^{u}}{\Lambda^{3}} \chi^{2} Q_{3}\left(\phi_{a}^{*} U_{a}\right) H \\
& +\frac{\lambda_{32}^{u}}{\Lambda} Q_{3}\left(\epsilon_{a b} \phi_{a} U_{b}\right) H+\lambda_{33}^{u} Q_{3} U_{3} H,
\end{aligned}
$$

and similar in the down and charged lepton sector. After inserting the spurion VEVs the cutoff dependence drops out, and Yukawa hierarchies arise from powers of the small parameters $\varepsilon_{\phi, \chi}$. In this way we get for the up-, down- and charged lepton Yukawa matrices (defined as $\mathcal{L}_{\text {yuk }}=Q^{T} Y_{u} U H+\cdots$ ) the result

$$
\begin{aligned}
Y_{u} & \approx\left(\begin{array}{ccc}
\lambda_{11}^{u} \varepsilon_{\phi}^{2} \varepsilon_{\chi}^{4} & \lambda_{12}^{u} \varepsilon_{\chi}^{2} & \lambda_{13}^{u} \varepsilon_{\phi} \varepsilon_{\chi}^{2} \\
-\lambda_{12}^{u} \varepsilon_{\chi}^{2} & \lambda_{22}^{u} \varepsilon_{\phi}^{2} & \lambda_{23}^{u} \varepsilon_{\phi} \\
\lambda_{31}^{u} \varepsilon_{\phi} \varepsilon_{\chi}^{2} & \lambda_{32}^{u} \varepsilon_{\phi} & \lambda_{33}^{u}
\end{array}\right), \quad Y_{d} \approx\left(\begin{array}{ccc}
\lambda_{11}^{d} \varepsilon_{\phi}^{2} \varepsilon_{\chi}^{4} & \lambda_{12}^{d} \varepsilon_{\chi}^{2} & \lambda_{13}^{d} \varepsilon_{\phi} \varepsilon_{\chi}^{3} \\
-\lambda_{12}^{d} \varepsilon_{\chi}^{2} & \lambda_{22}^{d} \varepsilon_{\phi}^{2} & \lambda_{23}^{d} \varepsilon_{\phi} \varepsilon_{\chi} \\
\lambda_{31}^{d} \varepsilon_{\phi} \varepsilon_{\chi}^{2} & \lambda_{32}^{d} \varepsilon_{\phi} & \lambda_{33}^{d} \varepsilon_{\chi}
\end{array}\right), \\
Y_{e} & \approx\left(\begin{array}{ccc}
\lambda_{11}^{e} \varepsilon_{\phi}^{2} \varepsilon_{\chi}^{4} & \lambda_{12}^{e} \varepsilon_{\chi}^{2} & \lambda_{13}^{e} \varepsilon_{\phi} \varepsilon_{\chi}^{2} \\
-\lambda_{12}^{e} \varepsilon_{\chi}^{2} & \lambda_{22}^{e} \varepsilon_{\phi}^{2} & \lambda_{23}^{e} \varepsilon_{\phi} \\
\lambda_{31}^{e} \varepsilon_{\phi} \varepsilon_{\chi}^{3} & \lambda_{32}^{e} \varepsilon_{\phi} \varepsilon_{\chi} & \lambda_{33}^{e} \varepsilon_{\chi}
\end{array}\right),
\end{aligned}
$$


where $\lambda_{i j}^{f}$ are (in general complex) $\mathcal{O}(1)$ coefficients and we have kept only the leading contributions in $\varepsilon_{\phi, \chi}$. Note that, in contrast to the supersymmetric U(2) model in ref. [6], there are no holomorphy constraints, which leads to a more general Yukawa pattern.

One can show that the $\lambda_{11}, \lambda_{13}, \lambda_{31}$ entries give only subleading corrections to quark masses and mixings, which are relatively suppressed by at least $\varepsilon_{\phi}^{2}$. Thus, effectively, three texture zeros appear in the Yukawa matrix, much as in the supersymmetric models [6], and to good approximation we obtain the Yukawa couplings

$$
\begin{aligned}
Y_{u} & \approx\left(\begin{array}{ccc}
0 & \lambda_{12}^{u} \varepsilon_{\chi}^{2} & 0 \\
-\lambda_{12}^{u} \varepsilon_{\chi}^{2} & \lambda_{22}^{u} \varepsilon_{\phi}^{2} & \lambda_{23}^{u} \varepsilon_{\phi} \\
0 & \lambda_{32}^{u} \varepsilon_{\phi} & \lambda_{33}^{u}
\end{array}\right), \quad Y_{d} \approx\left(\begin{array}{ccc}
0 & \lambda_{12}^{d} \varepsilon_{\chi}^{2} & 0 \\
-\lambda_{12}^{d} \varepsilon_{\chi}^{2} & \lambda_{22}^{d} \varepsilon_{\phi}^{2} & \lambda_{23}^{d} \varepsilon_{\phi} \varepsilon_{\chi} \\
0 & \lambda_{32}^{d} \varepsilon_{\phi} & \lambda_{33}^{d} \varepsilon_{\chi}
\end{array}\right), \\
Y_{e} & \approx\left(\begin{array}{ccc}
0 & \lambda_{12}^{e} \varepsilon_{\chi}^{2} & 0 \\
-\lambda_{12}^{e} \varepsilon_{\chi}^{2} & \lambda_{22}^{e} \varepsilon_{\phi}^{2} & \lambda_{23}^{e} \varepsilon_{\phi} \\
0 & \lambda_{32}^{e} \varepsilon_{\phi} \varepsilon_{\chi} & \lambda_{33}^{e} \varepsilon_{\chi}
\end{array}\right) .
\end{aligned}
$$

Because of the hierarchical structure and the presence of the texture zeros, it is possible to analytically derive some approximate results for the singular values and the rotations to the mass basis [7]. One can also perturbatively diagonalize the Yukawa matrices, and obtain the following estimates for singular values and CKM matrix elements (neglecting $\mathcal{O}(1)$ coefficients):

$$
\begin{aligned}
& y_{u} \sim \varepsilon_{\chi}^{4} / \varepsilon_{\phi}^{2}, \quad y_{d} \sim y_{e} \sim \varepsilon_{\chi}^{4} / \varepsilon_{\phi}^{2}, \quad V_{u b} \sim \varepsilon_{\chi}^{2} / \varepsilon_{\phi}, \\
& y_{c} \sim \varepsilon_{\phi}^{2}, \quad y_{s} \sim y_{\mu} \sim \varepsilon_{\phi}^{2} \varepsilon_{\chi} / \sqrt{\varepsilon_{\phi}^{2}+\varepsilon_{\chi}^{2}}, \quad V_{c b} \sim \varepsilon_{\phi} \text {, } \\
& y_{t} \sim 1, \quad y_{b} \sim y_{\tau} \sim \sqrt{\varepsilon_{\phi}^{2}+\varepsilon_{\chi}^{2}}, \quad V_{u s} \sim \varepsilon_{\chi}^{2} / \varepsilon_{\phi}^{2} .
\end{aligned}
$$

These expressions can be compared to the $(1 \sigma)$ ranges for fermion mass ratios and CKM elements, taken for definiteness at $10 \mathrm{TeV}$

$$
\begin{aligned}
& \frac{m_{u}}{m_{t}} \approx \lambda^{(7.1 \div 7.7)}, \quad \frac{m_{d}}{m_{b}} \approx \lambda^{(4.2 \div 4.4)}, \quad \frac{m_{e}}{m_{\tau}} \approx \lambda^{5.1}, \quad V_{u b} \approx \lambda^{3} \\
& \frac{m_{c}}{m_{t}} \approx \lambda^{3.5}, \quad \frac{m_{s}}{m_{b}} \approx \lambda^{(2.4 \div 2.5)}, \quad \frac{m_{\mu}}{m_{\tau}} \approx \lambda^{1.8}, \quad V_{c b} \approx \lambda^{2},
\end{aligned}
$$

where $\lambda=0.2 \approx V_{u s}$ and $y_{b}(10 \mathrm{TeV}) \approx \lambda^{2.7}, y_{\tau}(10 \mathrm{TeV}) \approx \lambda^{2.8}$. Within roughly a factor $\lambda$, all hierarchies can be reproduced taking

$$
\varepsilon_{\phi} \sim V_{c b} \sim \lambda^{2}, \quad \varepsilon_{\chi} \sim \lambda^{2 \div 3},
$$

and therefore a good fit to masses and mixings can be expected with input parameters $\lambda_{i j}^{f}$ that are indeed $\mathcal{O}(1)$. Moreover, it is clear that there must be four relations in each fermion sector between the 3 singular values and the $3+3$ rotation angles. For real $h_{i j}^{f}$ it is straightforward to work out these predictions exactly [7] and expand the result in ratios 
of the hierarchical eigenvalues. One can then relate the 1-2 and 1-3 rotations in the leftand right-handed sectors to the $2-3$ rotations and the eigenvalues. With the convention

$$
Y=V_{L} Y_{\text {diag }} V_{R}^{\dagger}, \quad V_{L}=V_{13}^{L} V_{12}^{L} V_{23}^{L}, \quad V_{R}=V_{13}^{R} V_{12}^{R} V_{23}^{R},
$$

where $V_{i j}$ are orthogonal rotation matrices in the $i-j$ plane that are parametrized by the angles $s_{i j} \equiv \sin \theta_{i j}$, one obtains up to percent corrections

$$
\begin{array}{lll}
s_{12}^{L u} \approx-s_{12}^{R u} \approx \sqrt{\frac{m_{u}}{m_{c}}}, & s_{13}^{L u} \approx-s_{23}^{L u} s_{12}^{L u}, & s_{13}^{R u} \approx s_{23}^{R u} s_{12}^{L u}, \\
s_{12}^{L d} \approx-s_{12}^{R d} \approx \sqrt{\frac{m_{d}}{m_{s}}} \sqrt{c_{23}^{R d}}, \quad s_{13}^{L d} \approx-s_{23}^{L d} s_{12}^{L d}\left(1-\frac{s_{23}^{R d}}{c_{23}^{R d} s_{23}^{L d}} \frac{m_{s}}{m_{b}}\right), & s_{13}^{R d} \approx \frac{s_{23}^{R d}}{c_{23}^{R d}} s_{12}^{L d}, \\
s_{12}^{R e} \approx-s_{12}^{L e} \approx \sqrt{\frac{m_{e}}{m_{\mu}}} \sqrt{c_{23}^{L e}}, & s_{13}^{R e} \approx-s_{23}^{R e} s_{12}^{R e}\left(1-\frac{s_{23}^{L e}}{c_{23}^{L e} s_{23}^{R e}} \frac{m_{\mu}}{m_{\tau}}\right), & s_{13}^{L e} \approx \frac{s_{23}^{L e}}{c_{23}^{L e}} s_{12}^{R e},
\end{array}
$$

where 2-3 rotations angles are large in the $\mathrm{RH}$ down and $\mathrm{LH}$ charged lepton sector, and CKM-like in all other sectors

$$
s_{23}^{R d} \sim s_{23}^{L e} \sim 1, \quad s_{23}^{L u} \sim s_{23}^{R u} \sim s_{23}^{L d} \sim s_{23}^{R e} \sim V_{c b} .
$$

One therefore obtains for the CKM elements (in our conventions $V_{\mathrm{CKM}}=V_{L}^{u T} V_{L}^{d *}$ ) the predictions

$$
\begin{array}{ll}
\left|V_{u b}\right| \approx\left|\sqrt{\frac{m_{u}}{m_{c}}}\right| V_{c b}\left|-e^{i \phi_{1}} \sqrt{\frac{m_{d}}{m_{s}}} \sqrt{c_{23}^{R d}} \frac{s_{23}^{R d}}{c_{23}^{R d}} \frac{m_{s}}{m_{b}}\right|, \quad\left|V_{t d}\right| \approx \sqrt{\frac{m_{d}}{m_{s}}} \sqrt{c_{23}^{R d}}|| V_{c b}\left|-e^{i \phi_{2}} \frac{s_{23}^{R d}}{c_{23}^{R d}} \frac{m_{s}}{m_{b}}\right|, \\
\left|V_{u s}\right| \approx\left|s_{12}^{L d}-s_{12}^{L u}\right| \approx\left|\sqrt{\frac{m_{d}}{m_{s}}} \sqrt{c_{23}^{R d}}-e^{i\left(\phi_{2}-\phi_{1}\right)} \sqrt{\frac{m_{u}}{m_{c}}}\right|, \quad\left|V_{c b}\right| \approx\left|V_{t s}\right| \approx\left|s_{23}^{L d}-s_{23}^{L u}\right|,
\end{array}
$$

where we included also relative phases $\phi_{1,2}$, see ref. [6] for details. In the original $\mathrm{U}(2)$ models in refs. [2, 3], the rotation angle in 2-3 RH down sector $s_{23}^{R d}$ was taken to be of the order of the other 2-3 rotation angles, $s_{23}^{R d} \sim V_{c b}$. From the above equations, this directly leads to the accurate prediction $\left|V_{u b} / V_{c b}\right| \approx \sqrt{m_{u} / m_{c}}$ which deviates from experimental data by more than $3 \sigma$. This is the reason why here this angle is taken to be large, $s_{23}^{R d} \sim c_{23}^{R d} \sim 1 / \sqrt{2}$, which then allows to obtain an excellent fit to CKM angles as we demonstrate in the next section (see also refs. $[4,6,7]$ ).

\subsection{Fit to quark and charged lepton sector}

We now perform a numerical fit to the model parameter set $\left\{\lambda_{i j}^{u, d, e}, \varepsilon_{\phi}, \varepsilon_{\chi}\right\}$. For simplicity, we restrict to real $\lambda_{i j}^{u, d, e}$ and demonstrate later on that the CKM phase can be obtained by taking a complex parameter $\lambda_{33}^{u}$. The experimental input parameters are therefore the quark and charged lepton masses and the CKM mixing angles. For concreteness we take them in the $\overline{\mathrm{MS}}$ scheme at $10 \mathrm{TeV}$ from ref. [13], with a symmetrized $1 \sigma$ error taken to be the larger one. All input parameters are summarized in table 2. The quality of the fit with a given model parameter set $\left\{\lambda_{i j}^{u, d, e}, \varepsilon_{\phi}, \varepsilon_{\chi}\right\}$ is measured by two functions $\chi^{2}$ and $\chi_{\mathcal{O}(1)}^{2}$. The first quantity is the usual $\chi^{2}$ that indicates how well the experimental input 


\begin{tabular}{|cc|}
\hline Quantity & Value \\
\hline$y_{u}$ & $(5.7 \pm 2.3) \times 10^{-6}$ \\
$y_{d}$ & $(1.223 \pm 0.180) \times 10^{-5}$ \\
$y_{s}$ & $(2.42 \pm 0.13) \times 10^{-4}$ \\
$y_{c}$ & $(2.776 \pm 0.088) \times 10^{-3}$ \\
$y_{b}$ & $(1.224 \pm 0.013) \times 10^{-2}$ \\
$y_{t}$ & $0.7894 \pm 0.0092$ \\
$y_{e}$ & $(2.8782 \pm 0.0042) \times 10^{-6}$ \\
$y_{\mu}$ & $(6.0761 \pm 0.0088) \times 10^{-4}$ \\
$y_{\tau}$ & $(1.0329 \pm 0.0015) \times 10^{-2}$ \\
\hline$\theta_{12}$ & $0.22736 \pm 0.00072$ \\
$\theta_{23}$ & $(4.364 \pm 0.067) \times 10^{-2}$ \\
$\theta_{13}$ & $(3.77 \pm 0.14) \times 10^{-3}$ \\
\hline
\end{tabular}

Table 2. Input values of quark and charged lepton Yukawas and quark mixing angles at $10 \mathrm{TeV}$ taken from ref. [13].

values are reproduced by the fit. It is obtained by plugging the model parameters into the Yukawa matrices in eq. (2.5) and calculating numerically the singular values $y_{q, l}$ and the CKM mixing angles $\theta_{i j}$ in the PDG parametrization. These values are used with the experimental input above to obtain $\chi^{2}$ defined as

$$
\chi^{2}=\sum_{q=u, d, s, c, b, t} \frac{\left(y_{q}-y_{q, \exp }\right)^{2}}{\left(\sigma y_{q, \exp }\right)^{2}}+\sum_{\ell=e, \mu, \tau} \frac{\left(y_{\ell}-y_{\ell, \exp }\right)^{2}}{\left(\sigma y_{\ell, \exp }\right)^{2}}+\sum_{(i j)=(12),(13),(23)} \frac{\left(\theta_{i j}-\theta_{i j, \exp }\right)^{2}}{\left(\sigma \theta_{i j, \exp }\right)^{2}} .
$$

In order to explain Yukawa hierarchies solely by $\mathrm{U}(2)_{F}$ breaking, the parameters $\lambda_{i j}^{u, d, e}$ should be $\mathcal{O}(1)$. The meaning of this requirement is somewhat fuzzy, and here we choose to quantify it by introducing a measure $\chi_{\mathcal{O}(1)}^{2}$ defined as

$$
\chi_{\mathcal{O}(1)}^{2}=\sum_{\lambda_{i j}^{p}} \frac{\left(\log \left(\left|\lambda_{i j}^{p}\right|\right)\right)^{2}}{2 \cdot 0.55^{2}}
$$

where $i, j=1,2,3$ and $p=u, d, e$. This corresponds to the assumption that the $\lambda_{i j}^{u, d, e}$ are distributed according to a log-normal distribution with mean 1 and standard deviation $\sigma=$ 0.55 , i.e. the absolute values $\lambda_{i j}^{u, d, e}$ lie with a probability of $95 \%$ within the interval $[1 / 3,3]$. For example, the contribution to $\chi_{\mathcal{O}(1)}^{2}$ of a single parameter $\lambda=\{3,5,7,10,50,100\}$ (or the inverse) is $\Delta \chi_{\mathcal{O}(1)}^{2}=\{2,4,6,9,25,35\}$. We consider a fit satisfactory as long as $\chi_{\mathcal{O}(1)}^{2} \leq$ \#pars, and there are 5 parameters for each fermion sector. As the best fit we choose the one that minimizes both $\chi^{2}$ and $\chi_{\mathcal{O}(1)}^{2}$.

In table 3 we show our fit results, where we display the values of the small parameters $\varepsilon_{\phi}, \varepsilon_{\chi}$ and indicate separately the two fit measures $\chi^{2}, \chi_{\mathcal{O}(1)}^{2}$ as defined above, along with the 


\begin{tabular}{|c|cccccc|}
\hline Fit & $\varepsilon_{\phi}$ & $\varepsilon_{\chi}$ & $\min \left|\lambda_{i j}^{u, d, \ell}\right|$ & $\max \left|\lambda_{i j}^{u, d, \ell}\right|$ & $\chi^{2}$ & $\chi_{\mathcal{O}(1)}^{2}$ \\
\hline $\mathrm{QL} 1_{\mathbb{R}}$ & 0.019 & 0.008 & $1 / 3.1$ & 2.7 & 1.7 & 7.8 \\
$\mathrm{QL} 2_{\mathbb{R}}$ & 0.023 & 0.008 & $1 / 2.7$ & 2.8 & 12 & 5.4 \\
\hline $\mathrm{QL} 3_{\mathbb{R}}$ & 0.065 & 0.011 & $1 / 9.1$ & 6.9 & 0 & 35 \\
\hline
\end{tabular}

Table 3. Best fits in the quark and charged lepton sector.

\begin{tabular}{|c|cccccc|}
\hline Fit & $\varepsilon_{\phi}$ & $\varepsilon_{\chi}$ & $\min \left|\lambda_{i j}^{u, d, \ell}\right|$ & $\max \left|\lambda_{i j}^{u, d, \ell}\right|$ & $\chi^{2}$ & $\chi_{\mathcal{O}(1)}^{2}$ \\
\hline QL1 & 0.025 & 0.009 & $1 / 2.9$ & 2.1 & 0.6 & 5.8 \\
QL2 & 0.024 & 0.008 & $1 / 2.8$ & 1.9 & 13 & 4.8 \\
\hline
\end{tabular}

Table 4. Best fits in the quark and charged lepton sector including the CKM phase.

smallest and largest $\left|\lambda_{i j}^{u, d, e}\right|$. For the fit $\mathrm{QL} 1_{\mathbb{R}}$ we have minimized $\chi^{2}+\chi_{\mathcal{O}(1)}^{2}$, while for $\mathrm{QL} 2_{\mathbb{R}}$ we have minimized $\chi_{\mathcal{O}(1)}^{2}$ while keeping $\chi^{2} \leq \#$ obs $=12$. For illustrative purposes we also show a fit that minimizes just $\chi^{2}\left(\mathrm{QL} 3_{\mathbb{R}}\right)$. Indeed there are enough free parameters to obtain a perfect fit to observables, however one needs $\chi_{\mathcal{O}(1)}^{2}$ as large as 35 and $\mathcal{O}(1)$ parameters as small as $\approx 1 / 9$, so this fit should be discarded according to our quality requirement $\chi_{\mathcal{O}(1)}^{2}<15$. The best fits are $\mathrm{QL} 1_{\mathbb{R}}$ and $\mathrm{QL} 2_{\mathbb{R}}$ with $\mathcal{O}(1)$ parameters between $1 / 3$ and 3 , which feature values of $\varepsilon_{\phi}, \varepsilon_{\chi}$ that are indeed of the naive size estimated in eq. (2.8).

Finally we demonstrate that the CKM phase $\delta_{\mathrm{CP}}$ can be easily included. For simplicity we restrict to the case where only the 33 entry in the up-quark Yukawa matrix is complex, i.e. $\lambda_{33}^{u} \rightarrow \lambda_{33}^{u} e^{i \delta_{33}}$. In a realistic setup where all Yukawas have phases, the fit can only get better. In the $\chi^{2}$ measure in eq. (2.13) we now include the CP phase of the CKM matrix, with the experimental value taken from ref. [13]

$$
\delta_{\mathrm{CP}, \exp }=1.208 \pm 0.054
$$

Including $\delta_{33}$ leads to even better fits (QL1 and QL2), which we show in table 4. This demonstrates that an excellent fit for quark and charged lepton sector, including the CKM phase, can be obtained with all $\mathcal{O}(1)$ parameters lying between $1 / 2.8$ and 2.1 .

\section{$2.3 \quad$ Neutrino sector}

In the neutrino sector we have to distinguish whether neutrinos are Dirac or Majorana. We begin with the discussion of the Dirac scenario, since the Majorana case in the $\mathrm{U}(2)_{F}$ model is strongly disfavored as we will discuss below. To this extent we introduce SM singlets $N_{a}, N_{3}$ with $\mathrm{U}(1)_{F}$ charges $X_{a}^{N}$ and $X_{3}^{N}$, where $N_{a}$ transforms as a doublet of $\mathrm{SU}(2)_{F}$ and $N_{3}$ as a singlet. The Lagrangian then allows for a Yukawa coupling $\mathcal{L}_{\nu}=$ $L^{T} Y_{\nu} N H$ (we assume that the Majorana mass term is forbidden, e.g. by exact lepton number conservation). As in the charged lepton sector, one can obtain its structure from 
a spurion analysis as

$$
Y_{\nu}=\left(\begin{array}{ccc}
\lambda_{11}^{\nu} \varepsilon_{\phi}^{2} \varepsilon_{\chi}^{\left|3+X_{a}^{N}\right|} & \lambda_{12}^{\nu} \varepsilon_{\chi}^{\left|1+X_{a}^{N}\right|} & \lambda_{13}^{\nu} \varepsilon_{\phi} \varepsilon_{\chi}^{\left|2+X_{3}^{N}\right|} \\
-\lambda_{12}^{\nu} \varepsilon_{\chi}^{\left|1+X_{a}^{N}\right|} & \lambda_{22}^{\nu} \varepsilon_{\phi}^{2} \varepsilon_{\chi}^{\left|X_{a}^{N}-1\right|} & \lambda_{23}^{\nu} \varepsilon_{\phi} \varepsilon_{\chi}^{\left|X_{3}^{N}\right|} \\
\lambda_{31}^{\nu} \varepsilon_{\phi} \varepsilon_{\chi}^{\left|2+X_{a}^{N}\right|} & \lambda_{32}^{\nu} \varepsilon_{\phi} \varepsilon_{\chi}^{\left|X_{a}^{N}\right|} & \lambda_{33}^{\nu} \varepsilon_{\chi}^{\left|1+X_{3}^{N}\right|}
\end{array}\right) .
$$

It is clear that in order to obtain sub-eV neutrinos one needs large $\mathrm{U}(1)_{F}$ charges $X_{a, 3}^{N}>1$, so that one can drop the absolute values in eq. (2.15). In this case the contributions from the (11), (13), (31) entries to masses and mixings are again sub-leading, and we can drop them as in the previous section and are left with the Dirac neutrino mass matrix

$$
m_{\nu}^{D} \approx v\left(\begin{array}{ccc}
0 & \lambda_{12}^{\nu} \varepsilon_{\chi}^{1+X_{a}^{N}} & 0 \\
-\lambda_{12}^{\nu} \varepsilon_{\chi}^{1+X_{a}^{N}} & \lambda_{22}^{\nu} \varepsilon_{\phi}^{2} \varepsilon_{\chi}^{X_{a}^{N}-1} & \lambda_{23}^{\nu} \varepsilon_{\phi} \varepsilon_{\chi}^{X_{3}^{N}} \\
0 & \lambda_{32}^{\nu} \varepsilon_{\phi} \varepsilon_{\chi}^{X_{a}^{N}} & \lambda_{33}^{\nu} \varepsilon_{\chi}^{1+X_{3}^{N}}
\end{array}\right) .
$$

It is well-known that an anarchical neutrino mass matrix can give a good fit to neutrino observables, which can be achieved taking $X_{a}^{N}=X_{3}^{N}$ (since $\varepsilon_{\chi} \sim \varepsilon_{\phi}$ ), giving

$$
m_{\nu}^{D} \approx v \varepsilon_{\chi}^{X_{a}^{N-1}}\left(\begin{array}{ccc}
0 & \lambda_{12}^{\nu} \varepsilon_{\chi}^{2} & 0 \\
-\lambda_{12}^{\nu} \varepsilon_{\chi}^{2} & \lambda_{22}^{\nu} \varepsilon_{\phi}^{2} & \lambda_{23}^{\nu} \varepsilon_{\phi} \varepsilon_{\chi} \\
0 & \lambda_{32}^{\nu} \varepsilon_{\phi} \varepsilon_{\chi} & \lambda_{33}^{\nu} \varepsilon_{\chi}^{2}
\end{array}\right)
$$

In order to obtain an overall neutrino mass scale $\lesssim 0.1 \mathrm{eV}$, one needs $X_{3}^{N} \gtrsim 5$, so that tiny neutrino masses arise from somewhat large $\mathrm{U}(1)_{F}$ charges and the smallness of the $\mathrm{U}(2)_{F}$ breaking parameters, $\varepsilon_{\chi, \phi} \sim 0.01$.

These considerations are confirmed by a numerical fit, for which we proceed as in the previous section, now including the neutrino sector. For the input values for normal (NO) and inverted mass ordering (IO), we use the neutrino mass differences and PMNS mixing angles from the global NuFIT $3.2(2018)$ in refs. [14, 15], which are summarized in table 5. We then plug the neutrino model parameters $\lambda_{i j}^{\nu}$ for fixed charges $X_{a}^{N}, X_{3}^{N}$ into the Yukawa matrices in eq. (2.17) and calculate numerically the singular values and the PMNS mixing angles $\theta_{i j}$ in the standard parametrization. To the $\chi^{2}$ defined in eq. (2.13) we add the corresponding expression $\chi_{\nu}^{2}$ in the neutrino sector ${ }^{1}$

$$
\chi_{\nu}^{2}=\sum_{(i j)=21,31 / 32} \frac{\left(\Delta m_{i j}^{2}-\Delta m_{i j, \exp }^{2}\right)^{2}}{\left(\sigma \Delta m_{i j, \exp }^{2}\right)^{2}}+\sum_{(i j)=(12),(13),(23)} \frac{\left(\sin ^{2} \theta_{i j}-\sin ^{2} \theta_{i j, \exp }\right)^{2}}{\left(\sigma \sin ^{2} \theta_{i j, \exp }\right)^{2}}
$$

and similarly we include the coefficients $\lambda_{i j}^{\nu}$ in the measure $\chi_{\mathcal{O}(1)}^{2}$ defined in eq. (2.14). We then perform a simultaneous fit to quark, charged lepton and neutrino sector including a phase in $\lambda_{33}^{u}$ as discussed in the last section (for simplicity we omit phases in the neutrino

\footnotetext{
${ }^{1}$ For the angle $\sin ^{2} \theta_{23}$ we actually use the full $\chi^{2}$ function provided by the NuFIT collaboration instead of assuming the Gaussian error in table 5 .
} 


\begin{tabular}{|c|c|}
\hline \multicolumn{2}{|c|}{ Normal Ordering (NO) } \\
\hline Quantity & Value \\
\hline$\Delta m_{21}^{2}$ & $(7.40 \pm 0.21) \times 10^{-5}$ \\
$\Delta m_{31}^{2}$ & $(2.494 \pm 0.033) \times 10^{-3}$ \\
\hline $\sin ^{2} \theta_{12}$ & $0.307 \pm 0.013$ \\
$\sin ^{2} \theta_{13}$ & $0.02206 \pm 0.00075$ \\
$\sin ^{2} \theta_{23}$ & $0.538 \pm 0.069$ \\
\hline
\end{tabular}

\begin{tabular}{|cc|}
\hline \multicolumn{2}{|c|}{ Inverted Ordering (IO) } \\
\hline Quantity & Value \\
\hline$\Delta m_{21}^{2}$ & $(7.40 \pm 0.21) \times 10^{-5}$ \\
$\Delta m_{32}^{2}$ & $(-2.465 \pm 0.032) \times 10^{-3}$ \\
\hline $\sin ^{2} \theta_{12}$ & $0.307 \pm 0.013$ \\
$\sin ^{2} \theta_{13}$ & $0.02227 \pm 0.00074$ \\
$\sin ^{2} \theta_{23}$ & $0.5540 \pm 0.0033$ \\
\hline
\end{tabular}

Table 5. Experimental values of neutrino mass differences and PMNS mixing angles for normal (NO) and inverted hierarchy (IO), taken from NuFIT $3.2(2018)[14,15]$.

\begin{tabular}{|c|cccccccc|}
\hline Fit & $X_{a}^{N}$ & $X_{3}^{N}$ & $\varepsilon_{\phi}$ & $\varepsilon_{\chi}$ & $\min \left|\lambda_{i j}^{u, d, e, \nu}\right|$ & $\max \left|\lambda_{i j}^{u, d, e, \nu}\right|$ & $\chi^{2}$ & $\chi_{\mathcal{O}(1)}^{2}$ \\
\hline $\mathrm{QL} \nu_{D}-1(\mathrm{NO})$ & 6 & 6 & 0.026 & 0.012 & $1 / 2.9$ & 2.6 & 0.5 & 10 \\
$\mathrm{QL} \nu_{D}-2(\mathrm{NO})$ & 6 & 6 & 0.024 & 0.013 & $1 / 2.6$ & 2.2 & 18 & 9 \\
$\mathrm{QL} \nu_{D}-3(\mathrm{NO})$ & 5 & 5 & 0.022 & 0.006 & $1 / 3.1$ & 3.8 & 1.0 & 13 \\
$\mathrm{QL} \nu_{D}-4(\mathrm{NO})$ & 5 & 5 & 0.021 & 0.006 & $1 / 2.5$ & 2.4 & 18 & 9 \\
\hline $\mathrm{QL} \nu_{D}(\mathrm{IO})$ & 6 & 6 & 0.015 & 0.013 & $1 / 9.1$ & 5.5 & 18 & 25 \\
\hline
\end{tabular}

Table 6. Best fits of the combined quark and lepton sector including CKM phase and Dirac neutrinos, with normal ordering (NO) or inverted ordering (IO). The complete set of parameters can be found in table 15 .

sector, including them would make the fit only better). The fit results are shown in table 6 , both for $\mathrm{NO}$ and IO. As expected, good fits are obtained only for equal charges $X_{a}^{N}=X_{3}^{N}=5 \div 6$. There is clearly a strong preference for NO, as can be seen in both quality parameters $\chi^{2}$ and $\chi_{\mathcal{O}(1)}^{2}$ (and the smallest/largest $\lambda_{i j}$ ). According to our quality requirement $\chi_{\mathcal{O}(1)}^{2}<20$, we should actually discard the IO possibility, since all fits with inverted mass ordering violate this criterion, and we include it just for illustrative purposes.

Comparing to the fit of quark and charged lepton sector only (cf. table 4), one can see that including neutrinos makes the fits slightly worse, but still with $\mathcal{O}(1)$ coefficients between $1 / 3$ and 3 . The fits determine all neutrino parameters, and we obtain predictions for the absolute mass scales and two important observables, the sum of masses $\Sigma m_{i}$ as probed by satellite telescopes, and the effective neutrino mass $m_{\beta}=\sqrt{\sum_{i} m_{i}^{2}\left|U_{e i}\right|^{2}}$ as measured in the $\beta$-decay spectrum close to the endpoint. All predictions are summarized in table 7. Since in contrast to the quark sector there are predictions for observables that are not yet measured, we also give a range for these predictions scanning over many fits with $X_{a}^{N}=X_{3}^{N}=5,6$ on which we only impose the (somewhat arbitrary) condition that $\chi^{2}<20$ and the quality requirement $\chi_{\mathcal{O}(1)}^{2}<20$ (which excludes IO). In this way we obtain predictions for the ranges of $\sum m_{i}$ and $m_{\beta}$ as shown in table 8 , where we also indicate the value preferred in most fits. We notice that the predicted range for $m_{\beta}$ is an order of magnitude below the expected future sensitivity of $m_{\beta} \lesssim 0.2 \mathrm{eV}$ by the KATRIN 


\begin{tabular}{|c|ccccc|}
\hline Fit & $m_{1}[\mathrm{meV}]$ & $m_{2}[\mathrm{meV}]$ & $m_{3}[\mathrm{meV}]$ & $\sum m_{i}[\mathrm{meV}]$ & $m_{\beta}[\mathrm{meV}]$ \\
\hline $\mathrm{QL} \nu_{D^{-1}}$ & 0.5 & 8.6 & 50 & 59 & 9 \\
$\mathrm{QL} \nu_{D^{-2}}$ & 4.6 & 9.6 & 50 & 64 & 10 \\
$\mathrm{QL} \nu_{D^{-3}}$ & 0.4 & 8.6 & 50 & 59 & 9 \\
$\mathrm{QL} \nu_{D^{-4}}$ & 0.4 & 8.6 & 50 & 59 & 9 \\
\hline
\end{tabular}

Table 7. Predictions for neutrino masses and observables for the NO fits in table 6 .

\begin{tabular}{|c|c|c|}
\hline Quantity & Range $[\mathrm{meV}]$ & Preferred values $[\mathrm{meV}]$ \\
\hline$\sum m_{i}$ & $58-110$ & $60-65$ \\
$m_{\beta}$ & $8-26$ & $9-10$ \\
\hline
\end{tabular}

Table 8. Range of predictions for $\sum m_{i}$ and $m_{\beta}$ scanning over fits with Dirac Neutrino charges $X_{a}^{N}=X_{3}^{N}=5,6$ and $\chi^{2}<20$ and $\chi_{\mathcal{O}(1)}^{2}<20$. In brackets indicated are the values preferred by most fits.

experiment [16]. The prediction for the neutrino mass sum is consistent with present bound by PLANCK giving $\sum m_{i}<0.12 \mathrm{eV}$ [17] and in the reach of the EUCLID satellite that is expected to measure $\sum m_{i}$ with an error of about $0.05 \mathrm{eV}[18,19]$. Note that the lower bound on the predicted range of $\sum m_{i}$ essentially saturates the minimal value that is obtained for a massless lightest neutrino, which (including $1 \sigma$ errors) is given by $58 \mathrm{meV}$ for normal ordering.

Finally, we discuss the case of Majorana Neutrinos. In addition to the neutrino Yukawa coupling, the Lagrangian contains a Majorana mass term, $\mathcal{L}_{\nu}=L^{T} Y_{\nu} N H+1 / 2 N^{T} M_{\nu} N+$ h.c. The Yukawa matrix $Y_{\nu}$ is the same as in eq. (2.15), while the Majorana mass matrix can be obtained as

$$
M_{\nu}=M\left(\begin{array}{ccc}
\kappa_{11} \varepsilon_{\phi}^{2} \varepsilon_{\chi}^{\left|2+2 X_{a}^{N}\right|} & \kappa_{12} \varepsilon_{\phi}^{2} \varepsilon_{\chi}^{\left|2 X_{a}^{N}\right|} & \kappa_{13} \varepsilon_{\phi} \varepsilon_{\chi}^{\left|1+X_{a}^{N}+X_{3}^{N}\right|} \\
\kappa_{12} \varepsilon_{\phi}^{2} \varepsilon_{\chi}^{\left|2 X_{a}^{N}\right|} & \kappa_{22} \varepsilon_{\phi}^{2} \varepsilon_{\chi}^{\left|2 X_{a}^{N}-2\right|} & \kappa_{23} \varepsilon_{\phi} \varepsilon_{\chi}^{\left|X_{a}^{N}+X_{3}^{N}-1\right|} \\
\kappa_{13} \varepsilon_{\phi} \varepsilon_{\chi}^{\left|1+X_{a}^{N}+X_{3}^{N}\right|} & \kappa_{23} \varepsilon_{\phi} \varepsilon_{\chi}^{\left|X_{a}^{N}+X_{3}^{N}-1\right|} & \kappa_{33} \varepsilon_{\chi}^{\left|2 X_{3}^{N}\right|}
\end{array}\right),
$$

where we factored out a single mass scale $M$ that is taken of the order of the usual see-saw scale, $M \sim 10^{14} \mathrm{GeV}$. One can therefore integrate out the heavy singlets and get light neutrino masses from the Weinberg operator $y_{i j} / M\left(L_{i} H\right)\left(L_{j} H\right)$, according to the type-I seesaw formula

$$
m_{\nu}^{M}=v^{2} Y_{\nu} M_{\nu}^{-1} Y_{\nu}^{T}
$$

Notice that the 1-2 entry of $M_{\nu}$ without any $\phi$ insertion vanishes because of the necessary $\mathrm{SU}(2)$ anti-symmetrization, and therefore picks up an additional $\varepsilon_{\phi}^{2}$ suppression. It turns out that this extra suppression spoils the naive EFT spurion analysis of the Weinberg operator using only the charges of $L_{a}, L_{3}$ (since negative powers of $\phi$ appear in the UV theory), and one has to use eq. (2.20) to calculate $m_{\nu}^{M}$. We first assume that $X_{A}^{N} \geq 1$ and $X_{3}^{N} \geq 0$, so that one can drop the absolute values and obtain for the parametric structure 
of the light neutrino mass matrix

$$
m_{\nu}^{M} \sim \frac{v^{2}}{M}\left(\begin{array}{ccc}
\varepsilon_{\chi}^{4} / \varepsilon_{\phi}^{2} & \varepsilon_{\chi}^{2} / \varepsilon_{\phi}^{2} & \varepsilon_{\chi}^{3} / \varepsilon_{\phi} \\
\varepsilon_{\chi}^{2} / \varepsilon_{\phi}^{2} & 1 / \varepsilon_{\phi}^{2} & \varepsilon_{\chi} / \varepsilon_{\phi} \\
\varepsilon_{\chi}^{3} / \varepsilon_{\phi} & \varepsilon_{\chi} / \varepsilon_{\phi} & \varepsilon_{\chi}^{2}
\end{array}\right) \sim\left(\begin{array}{ccc}
\varepsilon^{2} & 1 & \varepsilon^{2} \\
1 & 1 / \varepsilon^{2} & 1 \\
\varepsilon^{2} & 1 & \varepsilon^{2}
\end{array}\right)
$$

where $\varepsilon \sim \varepsilon_{\phi} \sim \varepsilon_{\chi}$ (notice that the charges $X_{a, 3}^{N}$ drop out). Such a structure is clearly ruled out, since it gives singular values $\left\{\varepsilon^{2}, \varepsilon^{2}, 1 / \varepsilon^{2}\right\}$, which would imply normal hierarchy along with a parametric prediction for the ratio of mass differences $\Delta m_{21}^{2} / \Delta m_{31}^{2} \sim \varepsilon^{4} \times \varepsilon^{4}$ that is way too small. Moreover, one can check that also different charge assignments for $X_{a, 3}^{N}$ do not allow to obtain a Majorana neutrino mass matrix that leads to a good fit, besides losing predictivity. Indeed the main theoretical advantage of Majorana neutrinos over Dirac neutrinos would be a scenario in which the effective Majorana mass matrix does not depend on the details of the UV physics, i.e. the choice of $X_{a, 3}^{N}$.

We conclude this section with the observation that the Majorana scenario would work perfectly if not for the vanishing of the leading 1-2 entry in the heavy mass matrix in eq. (2.19). Indeed, if this entry would be given by $\kappa_{12} \varepsilon_{\chi}^{\left|2 X_{a}^{N}\right|}$, and $X_{a}^{N} \geq 1, X_{3}^{N} \geq 0$, the effective light neutrino mass matrix would be given by (the dependence on $X_{a, 3}^{N}$ drops out again)

$$
m_{\nu}^{M} \sim \frac{v^{2}}{M}\left(\begin{array}{ccc}
\varepsilon_{\chi}^{4} \varepsilon_{\phi}^{2} & \varepsilon_{\chi}^{2} & \varepsilon_{\chi}^{3} \varepsilon_{\phi} \\
\varepsilon_{\chi}^{2} & \varepsilon_{\phi}^{2} & \varepsilon_{\chi} \varepsilon_{\phi} \\
\varepsilon_{\chi}^{3} \varepsilon_{\phi} & \varepsilon_{\chi} \varepsilon_{\phi} & \varepsilon_{\chi}^{2}
\end{array}\right) \sim\left(\begin{array}{ccc}
\varepsilon^{6} & \varepsilon^{2} & \varepsilon^{4} \\
\varepsilon^{2} & \varepsilon^{2} & \varepsilon^{2} \\
\varepsilon^{4} & \varepsilon^{2} & \varepsilon^{2}
\end{array}\right)
$$

which apart from the subleading $11,13,31$ entries has only very mild $\varepsilon_{\chi} / \varepsilon_{\phi}$ hierarchies and suggests a very good fit to neutrino observables. Note this absence of hierarchies is actually a prediction of the quark and charged lepton sector, which requires equal charges for the left-handed doublets $L_{a}$ and $L_{3}$, and order parameters of similar size, $\varepsilon_{\chi} \sim \varepsilon_{\phi}$. If therefore the 1-2 elements were symmetric instead of anti-symmetric, all low-energy mass matrices would follow the same hierarchical pattern, differing only in the $\mathrm{U}(1)_{F}$ charge assignment of the third generation, which is 0 for $Q_{3}, U_{3}, E_{3}$ and 1 for $D_{3}, L_{3}$. Thus the light $2 \times 2$ sub-block would be the same in all fermion sectors, and only the third coloum/row would differ by powers of $\varepsilon_{\chi}$, giving

$$
m_{\{u, d, e, \nu\}} \sim\left(\begin{array}{ccc}
0 & \varepsilon^{2} & 0 \\
\varepsilon^{2} & \varepsilon^{2} & \left\{\varepsilon, \varepsilon^{2}, \varepsilon, \varepsilon^{2}\right\} \\
0 & \left\{\varepsilon, \varepsilon, \varepsilon^{2}, \varepsilon^{2}\right\} & \left\{1, \varepsilon, \varepsilon, \varepsilon^{2}\right\}
\end{array}\right),
$$

where we neglected the mild $\varepsilon_{\chi} / \varepsilon_{\phi}$ hierarchy that is responsible for e.g. the Cabibbo angle. As we discuss in the following section, this simple pattern allows for an excellent fit to all fermion observables, and the necessary 1-2 symmetric structure can be obtained when considering the (discrete) dihedral group $D_{6}$ instead of $\mathrm{SU}(2)$ as flavor symmetry, which closely resembles the $\mathrm{SU}(2)$ structure apart from a sign flip in the 1-2 entries. 


\section{A $D_{6} \times \mathrm{U}(1)$ model of flavor}

In this section we consider the same framework with a $D_{6} \times \mathrm{U}(1)$ flavor symmetry, which closely resembles the $\mathrm{U}(2)$ case. We first introduce some $D_{6} \simeq D_{3} \times Z_{2}$ group theory and discuss the resulting flavor structure of quark and charged lepton masses, as well as the Weinberg operator. After some brief analytical considerations for the resulting predictions for neutrino observables, we perform a numerical fit to all fermion observables and conclude with a discussion of the phenomenological implications.

\subsection{Setup}

As we have just discussed, we want to mimic the structure of $\mathrm{U}(2)$ within a discrete flavor group that allows for a symmetric singlet contraction of two doublets. The simplest such group is the dihedral group $D_{3}$, the symmetry group of an equilateral triangle, which is discussed in detail in appendix A. This group is actually a subgroup of $\mathrm{SO}(3)$ and not of its double cover $\mathrm{SU}(2)$, and it is isomorphic to the permutation group $S_{3}$. It features two one-dimensional representations $\mathbf{1}$ and $\mathbf{1}^{\prime}$ and one two-dimensional representation $\mathbf{2}$. The contraction of two doublets $\psi=\left(\psi_{1}, \psi_{2}\right)$ and $\phi=\left(\phi_{1}, \phi_{2}\right)$ into the singlet $\mathbf{1}$ is given by

$$
(\psi \otimes \phi)_{1}=\psi_{1} \phi_{2}+\psi_{2} \phi_{1}
$$

Therefore we could simply assign the SM and spurion fields to $D_{3}$ representations that follow the $\mathrm{SU}(2)$ ones, i.e. the doublets $\mathbf{1 0}_{a}, \overline{\mathbf{5}}_{a}, \phi_{a}$ are in a $\mathbf{2}$ of $D_{3}$ and all other fields are total singlets. However, in contrast to $\mathrm{SU}(2)$ the product of two doublets also containts a doublet, so that three doublets can be contracted to a singlet as

$$
(\psi \otimes \phi \otimes \chi)_{1}=\psi_{1} \phi_{1} \chi_{1}+\psi_{2} \phi_{2} \chi_{2}
$$

This implies that in contrast to the $\mathrm{SU}(2)$ model a large 1-1 entry is generated, for example in the up-sector by the operator

$$
\mathcal{L} \supset \frac{1}{\Lambda^{2}}\left(\phi \otimes Q_{a} \otimes U_{a}\right)_{1} H \chi=\frac{1}{\Lambda^{2}}\left(\phi_{1} Q_{1} U_{1}+\phi_{2} Q_{2} U_{2}\right) H \chi=\varepsilon_{\phi} \varepsilon_{\chi} Q_{1} U_{1} H,
$$

which would be no longer negligible and thus would completely spoil the hierachical structure. In order to suppress this entry, we would like to mimic the $\mathrm{SU}(2)$ structure in which such a contraction is forbidden by the $Z_{2}$ center of $\mathrm{SU}(2)$, under which the doublets are odd and the singlet is even. Therefore, we consider ${ }^{2} D_{3} \times Z_{2}$ which is isomorphic to $D_{6}$, the symmetry group of a regular hexagon (see appendix A for details), and finally make the charge assignment as in table 9 . The additional $Z_{2}$ factor ensures that the contraction of three $\mathbf{2}_{-}$doublets does not contain the total singlet $\mathbf{1}_{+}$, and in the quark and charged lepton sector we obtain the very same spurion analysis as for $\mathrm{U}(2)$ in section 2 (see eq. (2.4)),

\footnotetext{
${ }^{2}$ Note we cannot use the double cover $\tilde{D}_{3}$ (which is an actual subgroup of $\mathrm{SU}(2)$ ) for this purpose, since that doublet $\mathbf{2}_{x}$ that contains no singlet in its cubic contraction, contains the singlet in its antisymmetric quadratic contraction.
} 


\begin{tabular}{|cccccccc|}
\hline & $\mathbf{1 0}_{a}$ & $\overline{\mathbf{5}}_{a}$ & $\mathbf{1 0}_{3}$ & $\overline{\mathbf{5}}_{3}$ & $H$ & $\phi_{a}$ & $\chi$ \\
\hline$D_{3} \times Z_{2}$ & $\mathbf{2}_{-}$ & $\mathbf{2}_{-}$ & $\mathbf{1}_{+}$ & $\mathbf{1}_{+}$ & $\mathbf{1}_{+}$ & $\mathbf{2}_{-}$ & $\mathbf{1}_{+}$ \\
$\mathrm{U}(1)_{F}$ & 1 & 1 & 0 & 1 & 0 & -1 & -1 \\
\hline
\end{tabular}

Table 9. The field content and $\left(D_{6} \simeq D_{3} \times Z_{2}\right) \times \mathrm{U}(1)_{F}$ quantum numbers.

except for the sign in the $1-2$ entry:

$$
\begin{aligned}
Y_{u} & \approx\left(\begin{array}{ccc}
\lambda_{11}^{u} \varepsilon_{\phi}^{2} \varepsilon_{\chi}^{4} & \lambda_{12}^{u} \varepsilon_{\chi}^{2} & \lambda_{13}^{u} \varepsilon_{\phi} \varepsilon_{\chi}^{2} \\
\lambda_{12}^{u} \varepsilon_{\chi}^{2} & \lambda_{22}^{u} \varepsilon_{\phi}^{2} & \lambda_{23}^{u} \varepsilon_{\phi} \\
\lambda_{31}^{u} \varepsilon_{\phi} \varepsilon_{\chi}^{2} & \lambda_{32}^{u} \varepsilon_{\phi} & \lambda_{33}^{u}
\end{array}\right), \quad Y_{d} \approx\left(\begin{array}{ccc}
\lambda_{11}^{d} \varepsilon_{\phi}^{2} \varepsilon_{\chi}^{4} & \lambda_{12}^{d} \varepsilon_{\chi}^{2} & \lambda_{13}^{d} \varepsilon_{\phi} \varepsilon_{\chi}^{3} \\
\lambda_{12}^{d} \varepsilon_{\chi}^{2} & \lambda_{22}^{d} \varepsilon_{\phi}^{2} & \lambda_{23}^{d} \varepsilon_{\phi} \varepsilon_{\chi} \\
\lambda_{31}^{d} \varepsilon_{\phi} \varepsilon_{\chi}^{2} & \lambda_{32}^{d} \varepsilon_{\phi} & \lambda_{33}^{d} \varepsilon_{\chi}
\end{array}\right), \\
Y_{e} & \approx\left(\begin{array}{ccc}
\lambda_{11}^{e} \varepsilon_{\phi}^{2} \varepsilon_{\chi}^{4} & \lambda_{12}^{e} \varepsilon_{\chi}^{2} & \lambda_{13}^{e} \varepsilon_{\phi} \varepsilon_{\chi}^{2} \\
\lambda_{12}^{e} \varepsilon_{\chi}^{2} & \lambda_{22}^{e} \varepsilon_{\phi}^{2} & \lambda_{23}^{e} \varepsilon_{\phi} \\
\lambda_{31}^{e} \varepsilon_{\phi} \varepsilon_{\chi}^{3} & \lambda_{32}^{e} \varepsilon_{\phi} \varepsilon_{\chi} & \lambda_{33}^{e} \varepsilon_{\chi}
\end{array}\right) .
\end{aligned}
$$

In the neutrino sector we work with the effective Weinberg operator $y_{i j} / M\left(L_{i} H\right)\left(L_{j} H\right)$, which can be induced by the type-I seesaw mechanism as discussed in the previous section. Its parametric structure is predicted in terms of the $D_{6} \times \mathrm{U}(1)_{F}$ quantum numbers of the charged leptons, which gives for the light Majorana neutrino mass matrix

$$
m_{\nu} \approx \frac{v^{2}}{M}\left(\begin{array}{ccc}
\lambda_{11}^{\nu} \varepsilon_{\chi}^{4} \varepsilon_{\phi}^{2} & \lambda_{12}^{\nu} \varepsilon_{\chi}^{2} & \lambda_{13}^{\nu} \varepsilon_{\phi} \varepsilon_{\chi}^{3} \\
\lambda_{12}^{\nu} \varepsilon_{\chi}^{2} & \lambda_{22}^{\nu} \varepsilon_{\phi}^{2} & \lambda_{23}^{\nu} \varepsilon_{\phi} \varepsilon_{\chi} \\
\lambda_{13}^{\nu} \varepsilon_{\phi} \varepsilon_{\chi}^{3} & \lambda_{23}^{\nu} \varepsilon_{\phi} \varepsilon_{\chi} & \lambda_{33}^{\nu} \varepsilon_{\chi}^{2}
\end{array}\right)
$$

Here we have used the same vacuum expectation values as before

$$
\langle\phi\rangle=\left(\begin{array}{c}
\varepsilon_{\phi} \Lambda \\
0
\end{array}\right), \quad\langle\chi\rangle=\varepsilon_{\chi} \Lambda,
$$

although in contrast to the $\mathrm{SU}(2)_{F}$ case we cannot use $D_{6}$ transformations in order to assume this VEV for $\phi$ without loss of generality. Therefore, we provide an explicit scalar potential in appendix $\mathrm{C}$ with only one additional scalar field that generates dynamically the above VEVs. ${ }^{3}$ Altogether, we obtain to good approximation the mass matrices

$$
\begin{array}{ll}
m_{u} \approx v\left(\begin{array}{ccc}
0 & \lambda_{12}^{u} \varepsilon_{\chi}^{2} & 0 \\
\lambda_{12}^{u} \varepsilon_{\chi}^{2} & \lambda_{22}^{u} \varepsilon_{\phi}^{2} & \lambda_{23}^{u} \varepsilon_{\phi} \\
0 & \lambda_{32}^{u} \varepsilon_{\phi} & \lambda_{33}^{u}
\end{array}\right), & m_{d} \approx v\left(\begin{array}{ccc}
0 & \lambda_{12}^{d} \varepsilon_{\chi}^{2} & 0 \\
\lambda_{12}^{d} \varepsilon_{\chi}^{2} & \lambda_{22}^{d} \varepsilon_{\phi}^{2} & \lambda_{23}^{d} \varepsilon_{\phi} \varepsilon_{\chi} \\
0 & \lambda_{32}^{d} \varepsilon_{\phi} & \lambda_{33}^{d} \varepsilon_{\chi}
\end{array}\right) \\
m_{e} \approx v\left(\begin{array}{cccc}
0 & \lambda_{12}^{e} \varepsilon_{\chi}^{2} & 0 \\
\lambda_{12}^{e} \varepsilon_{\chi}^{2} & \lambda_{22}^{e} \varepsilon_{\phi}^{2} & \lambda_{23}^{e} \varepsilon_{\phi} \\
0 & \lambda_{32}^{e} \varepsilon_{\phi} \varepsilon_{\chi} & \lambda_{33}^{e} \varepsilon_{\chi}
\end{array}\right), & m_{\nu} \approx \frac{v^{2}}{M}\left(\begin{array}{ccc}
0 & \lambda_{12}^{\nu} \varepsilon_{\chi}^{2} & 0 \\
\lambda_{12}^{\nu} \varepsilon_{\chi}^{2} & \lambda_{22}^{\nu} \varepsilon_{\phi}^{2} & \lambda_{23}^{\nu} \varepsilon_{\phi} \varepsilon_{\chi} \\
0 & \lambda_{23}^{\nu} \varepsilon_{\phi} \varepsilon_{\chi} & \lambda_{33}^{\nu} \varepsilon_{\chi}^{2}
\end{array}\right) .
\end{array}
$$

\footnotetext{
${ }^{3}$ Also a tiny VEV along the lower component of $\phi$ is generated, which however is small enough to give only negligible contributions to masses and mixings.
} 
As discussed in the previous section, this model has the remarkable feature that the hierarchies in the quark and charged lepton sector require $\varepsilon_{\phi} \sim \varepsilon_{\chi}$, and therefore naturally gives rise to an approximately anarchic neutrino mass matrix with generically large mixing angles.

Before we perform a numerical fit, we proceed with some analytical considerations. In the quark and charged lepton sector the analysis of the previous section is unaltered, since the flipped sign in the 1-2 entry does not play a role at leading order. In the neutrino sector we have 4 real parameters, which will enter the PMNS matrix together with three charged lepton rotations angles controlled by a single free real parameter $s_{23}^{L e}$, see eq. (2.10). These parameters correspond to 5 observables (3 PMNS angles +2 squared mass differences), so up to phases all parameters are fixed and one can predict the absolute neutrino mass scales and related observables. There are 4 phases in the neutrino sector and 2 phases in the left-handed charged lepton rotations, which combine to 3 physical phases, one Dirac and two Majorana phases. To study the prediction of the overall neutrino mass scale, we parametrize the neutrino mixing matrix $V_{\nu}$ (defined by $V_{\nu}^{T} m_{\nu} V=m_{\nu}^{\text {diag }}$ ) in the standard CKM form multiplied with a phase matrix $P_{\nu}=\operatorname{diag}\left(e^{i \alpha_{1}}, e^{i \alpha_{2}}, 1\right)$ from the right and a phase matrix $P^{\prime}$ from the left. Inverting the defining equation, we get from the vanishing 11 and 13 entries the two equations

$$
\begin{array}{r}
c_{12, \nu}^{2} \frac{m_{1}}{m_{3}} e^{-2 i\left(\alpha_{1}+\delta_{\nu}\right)}+s_{12, \nu}^{2} \frac{m_{2}}{m_{3}} e^{-2 i\left(\alpha_{2}+\delta_{\nu}\right)}+\frac{s_{13, \nu}^{2}}{c_{13, \nu}^{2}}=0, \\
\frac{m_{1}}{m_{3}} e^{-i\left(2 \alpha_{1}+\delta_{\nu}\right)}-\frac{m_{2}}{m_{3}} e^{-i\left(2 \alpha_{2}+\delta_{\nu}\right)}+\frac{s_{13, \nu} c_{23, \nu}}{c_{13, \nu}^{2} c_{12, \nu} s_{12, \nu} s_{23, \nu}}=0 .
\end{array}
$$

This leads to the inequalities

$$
\begin{aligned}
\left|1-\frac{c_{12, \nu}^{2}}{s_{12, \nu}^{2}} \frac{m_{1}}{m_{2}}\right| & \leq \frac{s_{13, \nu}^{2}}{s_{12, \nu}^{2} c_{13, \nu}^{2}} \frac{m_{3}}{m_{2}} \leq 1+\frac{c_{12, \nu}^{2}}{s_{12, \nu}^{2}} \frac{m_{1}}{m_{2}}, \\
1-\frac{m_{1}}{m_{2}} & \leq \frac{s_{13, \nu} c_{23, \nu}}{c_{13, \nu}^{2} c_{12, \nu} s_{12, \nu} s_{23, \nu}} \frac{m_{3}}{m_{2}} \leq 1+\frac{m_{1}}{m_{2}} .
\end{aligned}
$$

The angles in the neutrino sector $s_{i j, \nu}$ are connected to the observed PMNS mixing angles through $V_{\mathrm{PMNS}}=\left(V_{L}^{e}\right)^{T} V_{\nu}$. Since the 1-2 rotation in the charged lepton sector is small, $\sim \sqrt{m_{e} / m_{\mu}} \approx 0.07$, we have to good approximation $s_{12, \nu} \approx s_{12}$, but 2-3 rotations in the charged lepton sector are large, so that both $\theta_{23}$ and $\theta_{13}$ generically receive large contributions from the charged lepton sector. Nevertheless one can easily verify that eq. (3.10) cannot be satisfied for inverted mass ordering, while for normal ordering one can obtain an upper bound on the lightest neutrino mass $m_{1}$, by maximizing the neutrino mixing angles $s_{12, \nu}$ and $s_{13, \nu}$ with a suitable choice of phases. If one neglects the charged lepton contribution to $s_{12}$, one can show that $m_{1} \leq 11 \mathrm{meV}$, which in turn leads to upper bounds $\sum m_{i} \leq 76 \mathrm{meV}, m_{\beta} \leq 14 \mathrm{meV}$ and $m_{\beta \beta} \leq 13 \mathrm{meV}$. This estimate is confirmed by the numerical analysis in the next section.

\section{$3.2 \quad$ Numerical fit}

We now perform a simultaneous fit to quark, charged lepton and neutrino sector including a phase in $\lambda_{33}^{u}$ as in the last section (for simplicity we omit phases in the neutrino sector, 


\begin{tabular}{|c|cccccc|c|}
\hline Fit & $\varepsilon_{\phi}$ & $\varepsilon_{\chi}$ & $\min \left|\lambda_{i j}^{\mathrm{u}, \mathrm{d}, \ell}\right|$ & $\max \left|\lambda_{i j}^{\mathrm{u}, \mathrm{d}, \ell}\right|$ & $\chi^{2}$ & $\chi_{\mathcal{O}(1)}^{2}$ & $M\left[10^{11} \mathrm{GeV}\right]$ \\
\hline $\mathrm{QL} \nu_{M}-1$ & 0.025 & 0.009 & $1 / 2.8$ & 2.1 & 0.7 & 7.9 & 4.1 \\
$\mathrm{QL} \nu_{M}-2$ & 0.024 & 0.009 & $1 / 2.6$ & 1.9 & 18 & 6.3 & 3.3 \\
\hline
\end{tabular}

Table 10. Best fits for the $D_{6} \times \mathrm{U}(1)$ model including CKM phase and Majorana neutrinos. The complete set of parameters can be found in table 15 .

\begin{tabular}{|c|cccccc|}
\hline Fit & $m_{1}[\mathrm{meV}]$ & $m_{2}[\mathrm{meV}]$ & $m_{3}[\mathrm{meV}]$ & $\sum m_{i}[\mathrm{meV}]$ & $m_{\beta}[\mathrm{meV}]$ & $m_{\beta \beta}^{\max }[\mathrm{meV}]$ \\
\hline $\mathrm{QL} \nu_{M}-1$ & 1.0 & 8.7 & 50 & 60 & 8.8 & 4.4 \\
$\mathrm{QL} \nu_{M^{-}}$ & 1.5 & 8.8 & 50 & 60 & 8.9 & 4.9 \\
\hline
\end{tabular}

Table 11. Predictions for neutrino masses and observables for the fits in table 10. Since the prediction for $m_{\beta \beta}$ strongly depends on possible phases in the PMNS matrix, here we display the maximal possible value $m_{\beta \beta}^{\max }$.

\begin{tabular}{|c|c|c|}
\hline Quantity & Range $[\mathrm{meV}]$ & Preferred values $[\mathrm{meV}]$ \\
\hline$\sum m_{i}$ & $59-78$ & 60,70 \\
$m_{\beta}$ & $8-15$ & $9-10,11-12$ \\
$m_{\beta \beta}^{\max }$ & $3-16$ & 5,9 \\
\hline
\end{tabular}

Table 12. Range of predictions for $\sum m_{i}, m_{\beta}$ and $m_{\beta \beta}$ scanning over fits with $\chi^{2}<20$ and $\chi_{\mathcal{O}(1)}^{2}<20$. The last column indicates the values preferred by most fits.

including them would make the fit only better). The fit results are shown in table 10, and include also the effective suppression scale $M$ of Weinberg operator, which is of the order of $10^{11} \mathrm{GeV}$. The fit is even better compared to Dirac Neutrinos (cf. table 6), with all $\mathcal{O}(1)$ parameters roughly between 0.4 and 2 .

The corresponding predictions for the neutrino masses $m_{i}$, its sum $\sum m_{i}$, the neutrino mass $m_{\beta}$ and the "effective Majorana mass" $m_{\beta \beta}=\left|\sum U_{e i}^{2} m_{i}\right|$ measured in neutrinoless double-beta decay are shown in table 11. As expected from the analytical considerations, only a normal hierarchy for the neutrino masses is viable. The predicted values for $\sum m_{i}$ and $m_{\beta}$ are similar to the ones in the Dirac Neutrino case (cf. table 7), while the effective Majorana mass is well below the expected sensitivities even in near future neutrinoless double-beta decay experiments [20]. Finally, we also give a range for the observables scanning over many fits on which we only impose that $\chi^{2}<20$ and $\chi_{\mathcal{O}(1)}^{2}<20$. In this way we obtain predictions for $\sum m_{i}, m_{\beta}$ and $m_{\beta \beta}$ lying in the ranges shown in table 12, where we also indicate the value preferred in most fits. This result agrees well with our estimate in the last section, where we have also included phases, so we expect the upper bounds on the mass scales to be approximately valid even when including phases in the numerical fit (the lower bounds again saturate the limit obtained from taking the lightest neutrino massless).

We conclude this section with a discussion of the phenomenological implications of our model. As we have seen, the flavor sector itself gives rise to quite narrow predictions for 
observables in the neutrino sector, which are however far below the present experimental sensitivities. In order to obtain other experimental signals, we have to rely on new lowenergy dynamics besides the SM. The natural candidate for such new degrees of freedom are the fields at the cut-off scale $\Lambda$, which we have not specified so far (in particular the radial components of the flavons $\phi$ and $\chi$ naturally get a mass at that scale). However, effects of these fields and other dynamics related to the UV completion are suppressed by powers of $1 / \Lambda$, and there is no reason that $\Lambda$ is sufficiently close to the electroweak scale in order to give rise to sizable deviations from the SM. Still, it would be interesting to consider an explicit UV completion of the present model to study the structure of these effects in detail.

Another option for light dynamics, which is essentially model-independent and wellmotivated, is provided by the pseudo-scalars in the flavon fields. If there is no explicit breaking of the $\mathrm{U}(2)_{F}$ symmetry, the associated Goldstone bosons are exactly massless, apart from a linear combination that can be identified with the QCD axion, which solves the strong $\mathrm{CP}$ problem and gets a mass from non-perturbative effects. The easiest way to get rid of the orthogonal massless Goldstones is replacing $\mathrm{SU}(2)_{F}$ by a discrete subgroup, which is another advantage of the $D_{6} \times \mathrm{U}(1)$ model discussed in this section. In this case there a single Goldstone boson associated with the $\mathrm{U}(1)_{F}$ factor that can naturally serve as the QCD axion, as we are going to discuss in the next section.

\section{The $\mathrm{U}(2)$ axiflavon}

As originally proposed in ref. [10], a Goldstone boson arising from the breaking of global flavor symmetries could play the role of the QCD axion. Indeed any Goldstone of a U(1) symmetry with a QCD anomaly will solve the strong CP problem, and one can demonstrate (see ref. [11]) that there is a non-zero $\mathrm{SU}(3)_{c} \times \mathrm{SU}(3)_{c} \times \mathrm{U}(1)_{F}$ anomaly in any flavor model where the determinants of up-down and down-quark mass matrices are controlled dominantly by the $\mathrm{U}(1)_{F}$ symmetry factor. In the present model this is indeed the case as $\operatorname{det} m_{u} \sim \varepsilon_{\chi}^{4}$ and $\operatorname{det} m_{d} \sim \varepsilon_{\chi}^{5}$, due to the presence of the approximate texture zeros, see eq. (3.8). Moreover, if also the determinant of the charged lepton mass matrix depends only on the $\mathrm{U}(1)_{F}$ breaking, the ratio of electromagnetic and color anomaly coefficients $E / N$ is expected to be a rational number close to $8 / 3$ [11]. In the present model the $\mathrm{U}(1)_{F}$ charge assignment is actually compatible with $\mathrm{SU}(5)$, so it is clear that we get exactly $E / N=8 / 3$, as in minimal DFSZ [21, 22] and KSVZ models [23, 24], and thus the same axion couplings to photons.

In this section we will calculate the axion couplings to photons and fermions, concentrating on the flavor-violating couplings to fermions, which follow from the hierarchical structure of fermion masses and mixings. In particular, axion couplings to nucleons and electrons are fixed in terms of the $\mathrm{U}(1)_{F}$ charges, while flavor-violating couplings to quarks and leptons are controlled by the unitary rotations that diagonalize the Yukawa matrices. Their parametric suppression is determined by the $\mathrm{U}(2)_{F}$ quantum numbers, and their numerical value by the fit to fermion masses and mixings. We then study the phenomenology of this axion, finding that the strongest constraints on the axion mass (or equivalently 
the $\mathrm{U}(1)_{F}$ breaking scale) come from astrophysical constraints (as in the minimal DFSZ and KSVZ models), since flavor-violating axion couplings to light quarks are strongly suppressed by the approximate $\mathrm{SU}(2)_{F}$ structure.

\subsection{Axion couplings}

We begin by identifying the axiflavon as the Goldstone boson arising from the spontaneous breaking of $\mathrm{U}(1)_{F}$ induced by the VEVs of $\phi$ and $\chi$. In general, the Goldstone is a linear combination of the phases $a_{i}$ of the scalar fields $\phi_{i}$ with charge $X_{i}$ and (real) VEV $V_{i}$, given by

$$
a=\sum_{i} \frac{X_{i} V_{i} a_{i}}{\sqrt{\sum X_{j}^{2} V_{j}^{2}}} .
$$

Thus, we find that $\chi$ and $\phi$ contain the Goldstone as (we ignore the radial mode)

$$
\chi=\varepsilon_{\chi} \Lambda e^{-i a(x) / \sqrt{2} V}, \quad \phi=\left(\begin{array}{c}
\varepsilon_{\phi} \Lambda \\
0
\end{array}\right) e^{-i a(x) / \sqrt{2} V},
$$

where we have defined the $\mathrm{U}(1)_{F}$ breaking scale $V \equiv \sqrt{\varepsilon_{\chi}^{2}+\varepsilon_{\phi}^{2}} \Lambda$.

The couplings of $a$ to fermions can be obtained by inserting the above expressions for $\chi$ and $\phi$ into the effective Yukawa Lagrangian given by eq. (2.3) for the up sector and the analogous terms in the down- and charged lepton sector. It is then convenient to change field basis by performing a $\mathrm{U}(1)_{F}$ transformation of the fermion fields

$$
f \rightarrow f e^{i X_{f} a(x) / \sqrt{2} V}
$$

which will remove the $a(x)$ dependence from the Yukawa sector, because of $\mathrm{U}(1)_{F}$ invariance. Since this transformation is anomalous, it will generate axion couplings to gauge field strengths, and since it is local it will modify fermion kinetic terms. The resulting couplings to gluon and photon fields strengths are given by

$$
\mathcal{L}_{\text {anom }}=N \frac{a(x)}{\sqrt{2} V} \frac{\alpha_{s}}{4 \pi} G_{\mu \nu} \tilde{G}^{\mu \nu}+E \frac{a(x)}{\sqrt{2} V} \frac{\alpha_{\mathrm{em}}}{4 \pi} F_{\mu \nu} \tilde{F}^{\mu \nu}
$$

with the dual field strength $\tilde{F}_{\mu \nu}=\frac{1}{2} \varepsilon_{\mu \nu \rho \sigma} F^{\rho \sigma}$ and the anomaly coefficients

$$
\begin{aligned}
N= & \frac{1}{2}\left(4 X_{10_{a}}+2 X_{10_{3}}+2 X_{10_{a}}+X_{10_{3}}+2 X_{\overline{5}_{a}}+X_{\overline{5}_{3}}\right)=9 / 2, \\
E= & \frac{5}{3}\left(2 X_{10_{a}}+X_{10_{3}}\right)+\frac{4}{3}\left(2 X_{10_{a}}+X_{10_{3}}\right)+\frac{1}{3}\left(2 X_{\overline{5}_{a}}+X_{\overline{5}_{3}}\right) \\
& +\left(2 X_{\overline{5}_{a}}+X_{\overline{5}_{3}}\right)+\left(2 X_{10_{a}}+X_{10_{3}}\right)=12 .
\end{aligned}
$$

Thus, we obtain $E / N=8 / 3$ exactly, which is just a consequence of the fact that the $\mathrm{U}(1)_{F}$ charge assignment is compatible with $\mathrm{SU}(5)$. The modification of fermion kinetic terms leads to axion-fermion couplings in the flavor interaction basis

$$
\mathcal{L}_{a}=-\frac{\partial_{\mu} a}{\sqrt{2} V} \sum_{f} f_{i}^{\dagger} \bar{\sigma}^{\mu} X_{f_{i}} f_{i}
$$


In the mass basis, defined as $m_{f}=V_{f L} m_{f}^{\text {diag }}\left(V_{f R}\right)^{\dagger}$ we have

$$
\mathcal{L}_{a}=-\frac{\partial_{\mu} a}{\sqrt{2} V} \sum_{f=u, d, e}\left[g_{f_{i} f_{j}}^{L} f_{i}^{\dagger} \bar{\sigma}^{\mu} f_{j}+g_{f_{i} f_{j}}^{R} f_{i}^{c \dagger} \bar{\sigma}^{\mu} f_{i}^{c}\right],
$$

with

$$
\begin{aligned}
& g_{f_{i} f_{j}}^{L}=\left(V_{f L}\right)_{k i} X_{f_{k}}\left(V_{f L}\right)_{k j}^{*}=X_{f_{a}} \delta_{i j}+\left(X_{f_{3}}-X_{f_{a}}\right)\left(V_{f L}\right)_{3 i}\left(V_{f L}\right)_{3 j}^{*}, \\
& g_{f_{i} f_{j}}^{R}=\left(V_{f R}\right)_{k i}^{*} X_{f_{k}^{c}}\left(V_{f R}\right)_{k j}=X_{f_{a}^{c}} \delta_{i j}+\left(X_{f_{3}^{c}}-X_{f_{a}^{c}}\right)\left(V_{f R}\right)_{3 i}^{*}\left(V_{f R}\right)_{3 j} .
\end{aligned}
$$

Finally we switch to Dirac spinor notation for the fermions and introduce $f_{a} \equiv V /(\sqrt{2} N)$ to match to the standard normalization for the anomalous couplings. These are given by

$$
\mathcal{L}_{\text {anom }}=\frac{a(x)}{f_{a}} \frac{\alpha_{s}}{8 \pi} G_{\mu \nu} \tilde{G}^{\mu \nu}+\frac{E}{N} \frac{a(x)}{f_{a}} \frac{\alpha_{\mathrm{em}}}{8 \pi} F_{\mu \nu} \tilde{F}^{\mu \nu},
$$

with $E / N=8 / 3$ in this model (and domain wall number $N_{\mathrm{DW}}=2 N=9$ ). The couplings to fermions are given by

$$
\mathcal{L}_{a}=\frac{\partial_{\mu} a}{2 f_{a}} \bar{f}_{i} \gamma^{\mu}\left[C_{f_{i} f_{j}}^{V}+C_{f_{i} f_{j}}^{A} \gamma_{5}\right] f_{j}
$$

with

$$
\begin{aligned}
& C_{f_{i} f_{j}}^{V}=\frac{-g_{f_{i} f_{j}}^{L}+g_{f_{j} f_{i}}^{R}}{2 N}=\frac{X_{f_{a}^{c}}-X_{f_{a}}}{2 N} \delta_{i j}+\frac{X_{f_{3}^{c}}-X_{f_{a}^{c}}}{2 N} \varepsilon_{R, i j}^{f}-\frac{X_{f_{3}}-X_{f_{a}}}{2 N} \varepsilon_{L, i j}^{f}, \\
& C_{f_{i} f_{j}}^{A}=\frac{g_{f_{i} f_{j}}^{L}+g_{f_{j} f_{i}}^{R}}{2 N}=\frac{X_{f_{a}^{c}}+X_{f_{a}}}{2 N} \delta_{i j}+\frac{X_{f_{3}^{c}}-X_{f_{a}^{c}}}{2 N} \varepsilon_{R, i j}^{f}+\frac{X_{f_{3}}-X_{f_{a}}}{2 N} \varepsilon_{L, i j}^{f},
\end{aligned}
$$

and the shorthand notation

$$
\varepsilon_{L, i j}^{f} \equiv\left(V_{L}^{f}\right)_{3 i}\left(V_{L}^{f}\right)_{3 j}^{*}, \quad \varepsilon_{R, i j}^{f} \equiv\left(V_{R}^{f}\right)_{3 i}\left(V_{R}^{f}\right)_{3 j}^{*} .
$$

Note that the diagonal elements of these parameters satisfy

$$
0 \leq \varepsilon_{L / R, i i}^{f} \leq 1, \quad \sum_{i} \varepsilon_{L / R, i i}^{f}=1 .
$$

While the above expressions are valid for any axion model with PQ charges that are universal for two fermion generations, ${ }^{4}$ in the present model these expressions simplify to

$$
\begin{aligned}
C_{u_{i} u_{j}}^{V} & =\frac{\varepsilon_{L, i j}^{u}-\varepsilon_{R, i j}^{u}}{9}, & C_{u_{i} u_{j}}^{A} & =\frac{2 \delta_{i j}-\varepsilon_{L, i j}^{u}-\varepsilon_{R, i j}^{u}}{9}, \\
C_{d_{i} d_{j}}^{V} & =\frac{\varepsilon_{L, i j}^{d}}{9}, & C_{d_{i} d_{j}}^{A} & =\frac{2 \delta_{i j}-\varepsilon_{L, i j}^{d}}{9}, \\
C_{e_{i} e_{j}}^{V} & =-\frac{\varepsilon_{R, i j}^{e}}{9}, & C_{e_{i} e_{j}}^{A} & =\frac{2 \delta_{i j}-\varepsilon_{R, i j}^{e}}{9} .
\end{aligned}
$$

\footnotetext{
${ }^{4}$ See ref. [25] for a recent example where this structure is realized within a generalized DFSZ model, and can be used to suppress the axion couplings to nucleons and electrons.
} 
Using the approximate expressions in eq. (2.10), the rotations have the parametric structure

$$
V_{L}^{u} \sim V_{R}^{u} \sim\left(\begin{array}{ccc}
1 & \lambda & \lambda^{7} \\
\lambda & 1 & \lambda^{2} \\
\lambda^{3} & \lambda^{2} & 1
\end{array}\right), \quad V_{L}^{d} \sim V_{R}^{e} \sim\left(\begin{array}{ccc}
1 & \lambda & \lambda^{3} \\
\lambda & 1 & \lambda^{2} \\
\lambda^{3} & \lambda^{2} & 1
\end{array}\right), \quad V_{R}^{d} \sim V_{L}^{e} \sim\left(\begin{array}{ccc}
1 & \lambda & \lambda^{5} \\
\lambda & 1 & 1 \\
\lambda & 1 & 1
\end{array}\right)
$$

so that all relevant $V_{3 i}$ are CKM-like, and we have

$$
\varepsilon_{L}^{u} \sim \varepsilon_{R}^{u} \sim \varepsilon_{L}^{d} \sim \varepsilon_{R}^{e} \sim\left(\begin{array}{ccc}
\lambda^{6} & \lambda^{5} & \lambda^{3} \\
\lambda^{5} & \lambda^{4} & \lambda^{2} \\
\lambda^{3} & \lambda^{2} & 1
\end{array}\right)
$$

Therefore, the diagonal axial couplings are to very good approxmation independent of the rotations, and we get, denoting $C_{f_{i}} \equiv C_{f_{i} f_{i}}^{A}$,

$$
C_{u}=C_{d}=C_{e}=C_{c}=C_{s}=C_{\mu}=\frac{2}{9}, \quad C_{t}=0, \quad C_{b}=C_{\tau}=\frac{1}{9} .
$$

The flavor-violating axion couplings are controlled by $\varepsilon_{i j}^{f}$, whose numerical values, beyond the parametric suppression given above, are known for a given fit to masses and mixings. Besides there is an overall suppression factor $1 / f_{a}$ that is proportional to the axion mass $m_{a}$, with the usual conversion factor for QCD axions as obtained from Chiral Perturbation Theory [26] and Lattice QCD [27]

$$
m_{a}=5.7 \mu \mathrm{eV}\left(\frac{10^{12} \mathrm{GeV}}{f_{a}}\right)
$$

\subsection{Axion phenomenology}

The most important constraints on fermion couplings of invisible (stable) axions (cf. eq. 4.12) are summarized as an upper bound on the quantity $\left(m_{a} /\right.$ coupling $)$ in the first column of table 13. These include flavor-violating $b-s$ transitions as tested in $B \rightarrow K a$ decays [28], flavor-violating $s-d$ transitions contributing to $K \rightarrow \pi a$ decays [29], lepton flavor-violating $\mu-e$ transitions contributing to $\mu \rightarrow e a$ [30] and $\mu \rightarrow e a \gamma$ decays [31, 32], (flavor-diagonal) axion-electron couplings bounded by the measurement of the WD luminosity function [33], and effective axion couplings to nucleons constrained from the burst duration of the SN 1987A neutrino signal [34]. We did not include bounds from e.g. flavorviolating tau decays [35], since they give much weaker constraints. We have further used the predictions of the axion couplings in our model to obtain an upper bound on $m_{a}$, or equivalently a lower bound on $f_{a}$, which is shown in table 13 for the fit $\mathrm{QL} \nu_{M^{-}}-1$ of the complete $D_{6} \times \mathrm{U}(1)$ model in table 10 (the result for the other fits are very similar). As a result of the strong CKM protection of $s-d$ transitions $C_{s d}^{V} \sim \lambda^{5}$, the main constraint on the model comes from astrophysics, similar to flavor-universal axion models. Since the bound from WD cooling and SN1987A are comparable, and the precise value of the latter is debated in the literature (see e.g. the recent discussion in ref. [36] which finds a constraint on $m_{a} / C$ roughly a factor 5 weaker than the PDG bound), we only take the constraint from WD cooling, giving a upper bound on the axion mass $m_{a}<14 \mathrm{meV}$. This translates 


\begin{tabular}{|c|c|cc|c|}
\hline Coupling & $m_{a}^{\max } / C[\mathrm{eV}]$ & $m_{a}^{\max , \mathrm{U}(2)}[\mathrm{eV}]$ & $f_{a}^{\min , \mathrm{U}(2)}[\mathrm{GeV}]$ & Constraint \\
\hline$C_{\mu e}$ & $2.1 \cdot 10^{-3}$ & 78 & $7.3 \cdot 10^{4}$ & $\mu \rightarrow e a[30]$ \\
$C_{b s}^{V}$ & $9.1 \cdot 10^{-2}$ & 16 & $3.6 \cdot 10^{5}$ & $B^{+} \rightarrow K^{+} a[28]$ \\
$C_{s d}^{V}$ & $1.7 \cdot 10^{-5}$ & 0.58 & $9.8 \cdot 10^{6}$ & $K^{+} \rightarrow \pi^{+} a[29]$ \\
$C_{e e}^{A}$ & $3.1 \cdot 10^{-3}$ & 0.014 & $4.1 \cdot 10^{8}$ & WD Cooling [33] \\
$C_{N}$ & $3.5 \cdot 10^{-3}$ & 0.0092 & $6.2 \cdot 10^{8}$ & SN1987A [34] \\
\hline
\end{tabular}

Table 13. Bounds on selected axion-fermion couplings; here $C_{\mu e} \equiv \sqrt{\left(C_{\mu e}^{V}\right)^{2}+\left(C_{\mu e}^{A}\right)^{2}}$ and $C_{N} \equiv$ $\sqrt{C_{p}^{2}+C_{n}^{2}}$ denotes the effective couplings to nucleons, with axion couplings to protons and neutrons $C_{p, n}$ defined analogously to the axial vector couplings in eq. (4.12). The second column denotes the model-independent upper bounds on the ratio of $m_{a} / C$, where $C$ denotes the respective coupling, while the third and fourth columns contain the upper (lower) bound on $m_{a}\left(f_{a}\right)$ in our model, using the numerical results for the couplings of section 3.2, where for explicitness we took the fit $\mathrm{QL} \nu_{M}-1$ (other fits give similar constraints).

into a lower bound on the cutoff $\Lambda>1.9 \cdot 10^{10} \mathrm{GeV}$. The predictions for the branching ratio of $K^{+} \rightarrow \pi^{+} a$ decays are given

$$
\mathrm{BR}\left(K^{+} \rightarrow \pi^{+} a\right)=4.3 \cdot 10^{-14}\left(\frac{m_{a}}{14 \mathrm{meV}}\right)^{2}
$$

which is far below the future sensitivity of NA62 [37, 38] given the constraint from WD cooling. This is in sharp constrast to the U(1) Axiflavon proposed in ref. [11] (see also ref. [12]), where the $d-s$ transition is only Cabibbo-suppressed, $C_{s d}^{V} \sim \lambda$, so that $K^{+} \rightarrow \pi^{+} a$ provides the strongest constraint on the axion mass.

The upper bound on $m_{a}<14 \mathrm{meV}$ implies that the axion is stable on cosmological scales. It is a remarkable feature of the QCD axion that it can also explain the observed Dark Matter (DM) abundance. One of the simplest scenarios is the misalignment mechanism [39-41], valid when $\mathrm{U}(1)_{F}$ is broken before inflation. ${ }^{5}$ At this stage the axion is essentially massless and takes a generic field value misaligned from the vacuum value by an angle $\theta$. Around the QCD phase transition the axion potential is generated, and the axion begins to oscillate around the minimum. The energy density stored in these oscillations can be approximately related to the present DM abundance as [42]

$$
\Omega_{\mathrm{DM}} h^{2} \approx 0.12\left(\frac{6 \mu \mathrm{eV}}{m_{a}}\right)^{1.165} \theta^{2}
$$

where $\theta \in[-\pi, \pi]$ is the initial misalignment angle. Thus for not too small values $\theta \gtrsim 0.1 \pi$, the natural window for axion DM is given by axion masses roughly between $(1 \div 40) \mu \mathrm{eV}$, which correspond to axion decay constants $f_{a} \sim\left(10^{11} \div 10^{13}\right) \mathrm{GeV}$ and a cutoff in the

\footnotetext{
${ }^{5}$ Also cosmological scenarios with post-inflationary $\mathrm{U}(1)_{F}$ breaking are viable, provided the presence of a suitable explicit breaking term to solve the domain wall problem arising from $N_{\mathrm{DW}}=9$. This is in contrast to the U(1) Axiflavon in ref. [11], where the upper bound on the axion mass from $K \rightarrow \pi a$ prevents to obtain the right amount of axion dark matter if $\mathrm{U}(1)_{F}$ is broken after inflation.
} 


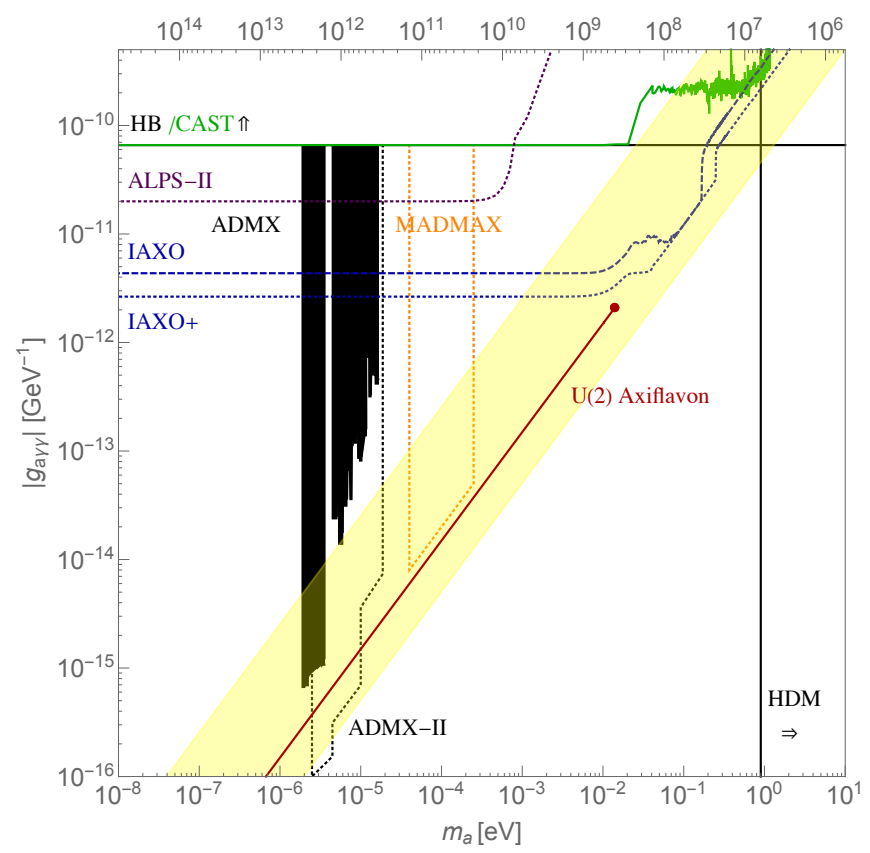

Figure 1. Prediction of the axion-photon coupling as a function of the axion mass $m_{a}$. The yellow band denotes the usual axion band of KSVZ models with a single pair of vector-like fermions, taken from ref. [44]. The red line denotes the parameter space of the U(2) Axiflavon model, which extend up to the $\bullet$ mark, denoting the bound from WD cooling, see table 13. Also shown are the bounds from structure formation excluding hot DM (HDM) [45-47], the bound from the evolution of Horizontal Branch (HB) stars in globular clusters [48], the expected sensitivity of the ALPS-II experiment [49], the present and future bounds from Axion helioscopes provided by CAST [50] and IAXO [51, 52], and from Axion Haloscopes like ADMX [53, 54], MADMAX [55] and the planned ADMX upgrade [43].

range $^{6} \Lambda \sim\left(10^{13} \div 10^{15}\right) \mathrm{GeV}$. This range of axion masses preferred by DM through the misalignment mechanism will be probed by the ADMX upgrade in the near future [43]. Indeed the discovery prospects of the $\mathrm{U}(2)$ Axiflavon are mainly due to its coupling to photons, and we summarize the status of the relevant experiments in the usual $\left(m_{a}, g_{a \gamma \gamma}\right)$ plane in figure 1 , where $g_{a \gamma \gamma}=|8 / 3-1.92| \alpha_{\mathrm{em}} /\left(2 \pi f_{a}\right)$.

\section{Summary and conclusions}

In summary, we have a proposed a $\mathrm{U}(2)_{F}$ model of flavor with horizontal quantum numbers compatible with an $\mathrm{SU}(5)$ GUT structure. The flavor symmetry $\mathrm{U}(2)_{F} \stackrel{\text { loc. }}{\simeq} \mathrm{SU}(2)_{F} \times$ $\mathrm{U}(1)_{F}$ is spontaneously broken by two flavon fields $\phi$ and $\chi$, which transform as a doublet and singlet under $\mathrm{SU}(2)_{F}$, respectively. Similarly, the three generations of SM fermions transform as $\mathbf{2}+\mathbf{1}$ of $\mathrm{SU}(2)_{F}$, and there is a simple assignment of $\mathrm{U}(1)_{F}$ quantum numbers

$$
X_{10_{3}}=0, \quad X_{10_{a}}=X_{\overline{5}_{a}}=X_{\overline{5}_{3}}=-X_{\phi}=-X_{\chi}=1 .
$$

\footnotetext{
${ }^{6}$ Repeating the numerical fit as in section 3.2 with SM input values at $10^{14} \mathrm{GeV}$, the $\chi^{2}$ and $\chi_{\mathcal{O}}^{2}(1)$ get slightly worse (0.4/11 and $18 / 9.1$ compared to $0.7 / 7.9$ and $18 / 6.3$ at $10 \mathrm{TeV}$, see table 10$)$, while the overall predictions change only marginally.
} 
The SM Yukawas arise from higher-dimensional operators made invariant under $\mathrm{U}(2)_{F}$ by appropriate insertions of flavons, suppressed by the cut-off scale $\Lambda \gg v$. In this way the hierarchical structure of Yukawa matrices is explained by powers of two small parameters that control the breaking of $\mathrm{U}(2)_{F}$, up to Wilson coefficients that are required to be $\mathcal{O}(1)$. The resulting Yukawa matrices in the quark and charged lepton sector have a simple structure with three texture zeros in the 1-1, 1-3 and 3-1 entries, while the 1-2 entry is antisymmetric, see eq. (2.5). The presence of these textures leads to accurate relations between CKM elements and masses, cf. eq. (2.12), which in contrast to the original $\mathrm{U}(2)$ flavor models in refs. $[2,3]$ can be consistent with experimental data because of large rotations in the right-handed down quark sector. Indeed we have obtained a very good fit to fermion masses and mixings with coefficients that are $\mathcal{O}(1)$ (all between 0.4 and 2), see table 4 .

We have then included the neutrino sector, which gives a consistent fit to experimental data only with Dirac neutrinos. To this extent, we have introduced three right-handed neutrinos (SM singlets), which also transform as $\mathbf{2}+\mathbf{1}$ of $\mathrm{SU}(2)_{F}$ and have equal charges under $\mathrm{U}(1)_{F}$. The resulting structure of the Dirac mass matrix (cf. eq. (2.17)) has again three texture zeros and only weak inter-generational hierarchies, thus predicting large mixing angles. The $\mathrm{U}(1)_{F}$ charge of the singlets enters only in the overall suppression factor and can account for the smallness of neutrino Yukawas if taken to be $5 \div 6$. The combined fit to the complete fermion sector is viable only for neutrinos with normal mass hierarchy, and still shows a good performance with $\mathcal{O}(1)$ coefficients between roughly $1 / 3$ and 3 (cf. table 6). This fit determines all parameters in the neutrino sector, and thus gives predictions for the absolute neutrino mass scale and the related observables. Scanning over many good fits we have obtained a range for the sum of neutrino masses roughly given by $(58 \div 110) \mathrm{meV}$, while the prediction for the effective neutrino mass measured in $\beta$-decays is far below future experimental sensitivities.

In order to have a consistent scenario with Majorana neutrinos, we have futhermore discussed an $D_{6} \times \mathrm{U}(1)$ variant of the $\mathrm{U}(2)_{F}$ model, where the $\mathrm{SU}(2)_{F}$ factor is replaced by a discrete $D_{6}$ subgroup. The charge assignment of fermions and spurions closely resembles the $\mathrm{U}(2)_{F}$ structure, so that the effective Yukawa matrices in the quark and charged lepton sector are exactly the same as in the $\mathrm{U}(2)_{F}$ case, up to a sign flip in the 1-2 entry that is largely irrelevant. This sign flip however allows for an unsuppressed 1-2 entry in the Weinberg operator, whose hierarchical structure follows directly from charges of the SM lepton doublets, and are to large extent independent of the charges of the heavy righthanded neutrinos (cf. eq. (2.22)). Remarkably, the resulting structure automatically leads to an anarchic neutrino mass matrix, so that the $\mathrm{SU}(5)$ structure connects large leptonic mixing angles to small mixing angles in the quark sector. Indeed, the parametric flavor suppression of up-, down-quark, charged lepton and neutrino masses follows the simple pattern

$$
m_{\{u, d, e, \nu\}} \sim\left(\begin{array}{ccc}
0 & \varepsilon^{2} & 0 \\
\varepsilon^{2} & \varepsilon^{2} & \left\{\varepsilon, \varepsilon^{2}, \varepsilon, \varepsilon^{2}\right\} \\
0 & \left\{\varepsilon, \varepsilon, \varepsilon^{2}, \varepsilon^{2}\right\} & \left\{1, \varepsilon, \varepsilon, \varepsilon^{2}\right\}
\end{array}\right)
$$


where the mass scale is set by $v$ in the quark and charged lepton sector and $v^{2} / M$ in the neutrino sector. The difference between the fermion sectors just follows from the different $\mathrm{U}(1)$ charge assignments for the third generation, see eq. (5.1). Although this model is more predictive than the Dirac case, since two U(1) charges are replaced by a single mass scale $M$, we obtain an excellent fit all SM observables with $\mathcal{O}(1)$ coefficients between 0.4 and 2 , see table 10 . From this fit we can again predict the overall neutrino mass scales, and as in the previous case only neutrinos with normal mass hierarchy are viable. Scanning over many good fits, we have obtained a slightly narrower range for the sum of neutrino masses roughly given by $(58 \div 78) \mathrm{meV}$, while again the predictions for the effective neutrino mass entering beta decay and neutrinoless double beta decay are far below future experimental sensitivities, see table 12 .

Finally we have discussed the various possibilities to test our models apart from the predictions in the neutrino sector. In general, sizable deviations in experimental observables from the SM require the existence of sufficiently light degrees of freedom. While there is no particular reason why the cutoff and its associated dynamics should be light, there is the natural possibility to solve the strong CP problem and account for DM through the Goldstone boson of the global $\mathrm{U}(1)_{F}$ symmetry, which we refer to as the U(2) Axiflavon. In contrast to the Axiflavon from a single Froggatt-Nielsen U(1) $F$ symmetry [56] as presented in refs. [11], here the flavor-violating couplings of the axion are protected by the approximate $\mathrm{U}(2)$ symmetry. Therefore, the $\mathrm{U}(2)$ Axiflavon looks very much like a usual DFSZ/KSVZ axion, with the strongest constraint from WD cooling, which requires a sufficiently light axion $m_{a}<14 \mathrm{meV}$. Particularly interesting is the axion mass range where DM can be explained through the misalignment mechanism, implying axion masses around $(1 \div 40) \mu \mathrm{eV}$, which corresponds to a cutoff scale of roughly $\left(10^{13} \div 10^{15}\right) \mathrm{GeV}$. This range will be tested by future axion haloscope searches.

The present model could be extended in several ways: 1) a more careful study of the neutrino sector might allow to pin down the predictions analytically, and it could be interesting to take a closer look to the type-I seesaw model, in particular its connection with Leptogenesis. 2) One could embed the model into a supersymmetric framework to address the hierarchy problem, possibly in connection with a full SU(5) GUT, trying to relate GUT breaking scale, flavor breaking scale and the axion decay constant, similar to ref. [57]. 3) Finally, it might be interesting to study possible UV completions and calculate the low-energy constraints from flavor-violating obervables on the new dynamics.

\section{Acknowledgments}

We thank F. Feruglio, J. Lopez Pavon, A. Ringwald, A. Romanino and A. Trautner for useful discussions and comments. ML acknowledges the support by the DFG-funded Doctoral School "Karlsruhe School of Elementary and Astroparticle Physics: Science and Technology". 


\begin{tabular}{|ccc|}
\hline Representation & $R$ & $S$ \\
\hline $\mathbf{1}$ & 1 & 1 \\
$\mathbf{1}^{\prime}$ & 1 & -1 \\
$\mathbf{2}$ & $\left(\begin{array}{cc}e^{\frac{2 \pi i}{3}} \\
e^{\frac{-2 \pi i}{3}}\end{array}\right)$ & $\left(\begin{array}{ll}0 & 1 \\
1 & 0\end{array}\right)$ \\
\hline
\end{tabular}

Table 14. Representation matrices for $D_{3}$.

\section{A $\quad D_{3}$ and $D_{6}$ group theory}

In this appendix we provide some details about the structure of the dihedral groups $D_{3}$ and $D_{6}$ and fix the notation for constructing group invariants (see also refs. $[5,58,59]$ ).

The dihedral group $D_{3}$ is the symmetry group of an equilateral triangle and is isomorphic to $S_{3}$, the permutation group of three objects with order 6 . The group is generated by two elements $R$ and $S$, where $R$ is the rotation through $120^{\circ}$ and $S$ is the reflection about one of the bisectors. Since $R^{3}=S^{2}=1$ and $S R=R^{2} S$, the six elements are $1, R, R^{2}, S, R S, S R$.

$D_{3}$ has two one-dimensional representations $\mathbf{1}, \mathbf{1}^{\prime}$ and one two-dimensional representation 2. The representation matrices for $R$ and $S$ can be chosen as in table 14 . The tensor products of two one-dimensional representations decompose as follows:

$$
1 \otimes 1=1, \quad 1 \otimes 1^{\prime}=1^{\prime}, \quad 1^{\prime} \otimes 1^{\prime}=1,
$$

while for the product of two 2's one gets

$$
\mathbf{2} \otimes \mathbf{2}=\mathbf{1} \oplus \mathbf{1}^{\prime} \oplus \mathbf{2} .
$$

For two doublets $\psi=\left(\begin{array}{l}\psi_{1} \\ \psi_{2}\end{array}\right)$ and $\varphi=\left(\begin{array}{l}\varphi_{1} \\ \varphi_{2}\end{array}\right)$ one finds

$$
(\psi \otimes \varphi)_{\mathbf{1}}=\psi_{1} \varphi_{2}+\psi_{2} \varphi_{1}, \quad(\psi \otimes \varphi)_{\mathbf{1}^{\prime}}=\psi_{1} \varphi_{2}-\psi_{2} \varphi_{1}, \quad(\psi \otimes \varphi)_{\mathbf{2}}=\left(\begin{array}{c}
\psi_{2} \varphi_{2} \\
\psi_{1} \varphi_{1}
\end{array}\right) .
$$

In the following we will use the simplified notation for singlet components (i.e. invariants)

$$
(\psi \cdot \varphi) \equiv(\psi \otimes \varphi)_{1}=\psi_{1} \varphi_{2}+\psi_{2} \varphi_{1} .
$$

From a given doublet $\varphi$ one can construct another doublet $\widetilde{\varphi}=\sigma^{1} \varphi^{*}=\left(\begin{array}{l}\varphi_{2}^{*} \\ \varphi_{1}^{*}\end{array}\right)$, with invariant

$$
(\widetilde{\varphi} \cdot \varphi)=\varphi_{1}^{*} \varphi_{1}+\varphi_{2}^{*} \varphi_{2} .
$$

Note that because of eq. (A.2) any product of doublets contain at least one singlet. For three doublets it is given by

$$
(\psi \cdot \varphi \cdot \chi)=\psi_{1} \varphi_{1} \chi_{1}+\psi_{2} \varphi_{2} \chi_{2}
$$




\begin{tabular}{|c|c|c|c|c|c|c|}
\hline Parameter & $\mathrm{QL} \nu_{D^{-1}}$ & $\mathrm{QL} \nu_{D^{-}} 2$ & $\mathrm{QL} \nu_{D^{-}} 3$ & $\mathrm{QL} \nu_{D^{-4}}$ & $\mathrm{QL} \nu_{M-1}$ & $\mathrm{QL} \nu_{M}-2$ \\
\hline$\lambda_{12}^{u}$ & 0.902 & 0.843 & 3.831 & 1.162 & -1.633 & -1.176 \\
\hline$\lambda_{22}^{u}$ & 1.187 & -1.047 & 1.859 & 1.148 & 1.339 & 1.112 \\
\hline$\lambda_{23}^{u}$ & 2.222 & -2.175 & -2.138 & -1.799 & 2.127 & 1.925 \\
\hline$\lambda_{32}^{u}$ & -1.103 & -1.419 & 1.511 & 2.422 & 1.196 & 1.615 \\
\hline$\lambda_{33}^{u}$ & 0.787 & 0.779 & -0.787 & 0.786 & 0.787 & 0.785 \\
\hline$\delta_{33}$ & -0.640 & -0.720 & -3.948 & -1.097 & -3.837 & -3.988 \\
\hline$\lambda_{12}^{d}$ & 0.479 & -0.479 & 2.165 & 2.173 & -0.888 & 0.976 \\
\hline$\lambda_{22}^{d}$ & -1.000 & -1.156 & -1.075 & -0.972 & -0.973 & 0.976 \\
\hline$\lambda_{23}^{d}$ & 0.913 & -0.786 & -1.304 & -1.155 & 1.073 & 0.985 \\
\hline$\lambda_{32}^{d}$ & -0.355 & 0.401 & 0.414 & 0.423 & 0.365 & -0.394 \\
\hline$\lambda_{33}^{d}$ & 0.665 & 0.651 & 1.394 & 1.497 & -0.902 & -0.948 \\
\hline$\lambda_{12}^{\ell}$ & 0.402 & -0.376 & -1.752 & -1.758 & -0.801 & 0.856 \\
\hline$\lambda_{22}^{\ell}$ & 0.987 & -1.134 & 1.821 & 2.052 & 1.306 & 1.497 \\
\hline$\lambda_{23}^{\ell}$ & 0.343 & 0.381 & 0.393 & -0.414 & -0.368 & 0.391 \\
\hline$\lambda_{32}^{\ell}$ & -0.992 & -1.132 & 1.175 & 1.193 & -1.198 & 1.294 \\
\hline$\lambda_{33}^{\ell}$ & 0.432 & -0.399 & -0.945 & 0.992 & -0.503 & -0.536 \\
\hline$\lambda_{12}^{\nu}$ & 0.882 & -1.416 & 0.938 & 1.006 & 2.130 & -1.873 \\
\hline$\lambda_{22}^{\nu}$ & -0.994 & -1.303 & 0.325 & 0.398 & -0.844 & -0.760 \\
\hline$\lambda_{23}^{\nu}$ & -2.588 & -1.074 & -1.505 & 1.681 & 1.137 & -1.078 \\
\hline$\lambda_{32}^{\nu}$ & 1.065 & -0.704 & 0.601 & 0.680 & 11 & II \\
\hline$\lambda_{33}^{\nu}$ & 0.952 & -1.572 & -0.890 & 0.891 & -0.489 & -0.655 \\
\hline$X_{a}^{\mathrm{N}}$ & 6 & 6 & 5 & 5 & & \\
\hline$X_{3}^{\mathrm{N}}$ & 6 & 6 & 5 & 5 & & \\
\hline$v / M \times 10^{9}$ & & & & & -0.421 & -0.520 \\
\hline$\varepsilon_{\phi}$ & 0.026 & 0.024 & 0.022 & 0.021 & 0.025 & 0.024 \\
\hline$\varepsilon_{\chi}$ & 0.012 & 0.013 & 0.006 & 0.006 & 0.009 & 0.009 \\
\hline
\end{tabular}

Table 15. Fit parameters for Dirac $(\mathrm{SU}(2) \times \mathrm{U}(1)$ Model $)$ and Majorana neutrinos $\left(D_{6} \times \mathrm{U}(1)\right.$ Model). The parameters are defined in eqs. (2.5) and (2.17), and eq. (3.8), respectively. 
while there are three different singlets in the product of four doublets, which we define as

$$
(\psi \otimes \varphi \otimes \chi \otimes \eta)_{1}=\left\{\begin{array}{l}
\psi_{1} \varphi_{2} \chi_{1} \eta_{2}+\psi_{2} \varphi_{1} \chi_{2} \eta_{1} \\
\psi_{1} \varphi_{2} \chi_{2} \eta_{1}+\psi_{2} \varphi_{1} \chi_{1} \eta_{2} \\
\psi_{1} \varphi_{1} \chi_{2} \eta_{2}+\psi_{2} \varphi_{2} \chi_{1} \eta_{1}
\end{array}\right.
$$

For the case of $\psi=\varphi$ and $\chi=\eta$ there are just two invariants for which we use the notation:

$$
(\psi \otimes \psi \otimes \chi \otimes \chi)_{\mathbf{1}}=\left\{\begin{array}{l}
(\psi \cdot \psi)(\chi \cdot \chi) \equiv 4 \psi_{1} \psi_{2} \chi_{1} \chi_{2} \\
(\psi \cdot \psi \cdot \chi \cdot \chi) \equiv \psi_{1}^{2} \chi_{2}^{2}+\psi_{2}^{2} \chi_{1}^{2}
\end{array}\right.
$$

Finally we turn to the dihedral group $D_{6}$ which is the symmetry group of regular hexagon. It is isomorphic to $D_{3} \times Z_{2}$, and therefore inherits the group theoretical structure discussed above, except that each representation carries an additional $Z_{2}$ charge, which is conserved in tensor decompositions. Thus, we have four one-dimensional representations $\mathbf{1}_{+}, \mathbf{1}_{-}, \mathbf{1}_{+}^{\prime}, \mathbf{1}_{-}^{\prime}$ (where $\mathbf{1}_{+}$denotes the total singlet) and two two-dimensional representations $\mathbf{2}_{+}, \mathbf{2}_{-}$. The decompositions of these representations follow from the $D_{3}$ ones, for example we have

$$
\mathbf{2}_{-} \otimes \mathbf{2}_{-}=\mathbf{1}_{+} \oplus \mathbf{1}_{+}^{\prime} \oplus \mathbf{2}_{+}, \quad \mathbf{2}_{+} \otimes \mathbf{2}_{-}=\mathbf{1}_{-} \oplus \mathbf{1}_{-}^{\prime} \oplus \mathbf{2}_{-} .
$$

Therefore in $D_{6}$ the tensor product $\left(\mathbf{2}_{-} \otimes \mathbf{2}_{-} \otimes \mathbf{2}_{-}\right)$does not contain a singlet.

\section{B Fit results}

Below we also provide the finetuning and pulls of the fit. For each observable $O_{i}=$ $\left\{y_{u}, y_{d}, \ldots\right\}$ we define the tuning $\Delta_{i}$ and the pull $P_{i}$ as

$$
\Delta_{i}=\max _{j}\left|\frac{\partial \log O_{i}}{\partial \log p_{j}}\right|, \quad P_{i}=\frac{O_{i}^{\mathrm{fit}}-O_{i}^{\exp }}{\sigma_{i}^{\exp }}
$$

where $p_{j}=\left\{\lambda_{i j}^{u, d, \ell, \nu}, \varepsilon_{\phi}, \varepsilon_{\chi}, M\right\}$ are the fit parameters. For the sake of brevity, we restrict to Fit 3 and 4 in the Dirac case, the other two fits give similar results. As can be seen from tables 16 and 17, the tuning of the observables is quite low, at most $10 \%$ for the Dirac case and about $20 \%$ in the Majorana case. As expected from the $\chi^{2}$ value, the pulls are small and are dominated by the quark Yukawas (and in the Majorana case also by the PMNS mixing angles).

\section{Scalar potential}

In this section we consider an explicit scalar potential that generates the VEVs we have assumed in section 3, serving merely as a proof of existence. In particular, this potential should be reassessed in a UV complete setup, possibly in connection with a supersymmetric $\mathrm{SU}(5)$ GUT.

In addition to the scalars $\phi$ and $\chi$ we need to introduce a new (SM singlet) scalar $\psi$ in order to break the $\mathrm{U}(1)$ symmetries in the scalar potential to a single continuous 


\begin{tabular}{|c|cc|cc|}
\hline Observable & $\Delta\left(\mathrm{QL} \nu_{D^{-}} 3\right)$ & $\mathrm{Pull}\left(\mathrm{QL} \nu_{D^{-}}\right)$ & $\Delta\left(\mathrm{QL} \nu_{D^{-}}\right)$ & $\mathrm{Pull}\left(\mathrm{QL} \nu_{D^{-}}\right)$ \\
\hline$y_{u}$ & 4 & 0.0 & 4 & -2.3 \\
$y_{c}$ & 2 & -0.1 & 2 & -1.2 \\
$y_{t}$ & 1 & -0.0 & 1 & -0.1 \\
$y_{d}$ & 3.8 & 0.9 & 3.8 & 2.1 \\
$y_{s}$ & 1.4 & -0.2 & 1.3 & -2.3 \\
$y_{b}$ & 0.6 & -0.0 & 0.5 & 0.1 \\
$y_{e}$ & 4 & -0.0 & 4 & -0.0 \\
$y_{\mu}$ & 1.3 & 0.0 & 1.3 & -0.0 \\
$y_{\tau}$ & 0.7 & 0.0 & 0.7 & 0.0 \\
$\theta_{13}^{\mathrm{CKM}}$ & 1.2 & -0.1 & 1.1 & -0.1 \\
$\theta_{12}^{\mathrm{CKM}}$ & 1.8 & -0.1 & 1.7 & -0.2 \\
$\theta_{23}^{\mathrm{CKM}}$ & 3.8 & -0.0 & 1.1 & 0.0 \\
$\delta_{\mathrm{CP}}$ & 4.8 & -0.0 & 0.8 & 0.6 \\
$\Delta m_{21}^{2}$ & 11.3 & -0.0 & 11.4 & -0.3 \\
$\Delta m_{31}^{2}$ & 9.3 & 0.0 & 9.3 & 0.3 \\
$\theta_{13}^{\text {PMNS }}$ & 1.3 & 0.1 & 1.3 & 0.6 \\
$\theta_{12}^{\text {PMNS }}$ & 1.1 & 0.0 & 1.1 & -0.5 \\
$\theta_{23}^{\text {PMNS }}$ & 0.7 & 0.0 & 0.7 & 0.7 \\
\hline
\end{tabular}

Table 16. Fine-tuning and pulls for the observables of fit $\mathrm{QL} \nu_{D^{-}} 3$ and fit $\mathrm{QL} \nu_{D^{-}} 4$.

global symmetry that can be identified with $\mathrm{U}(1)_{F}$. The transformation properties under $D_{6}\left[\mathrm{U}(1)_{F}\right]$ are

$$
\phi=2_{-}[-1], \quad \chi=1_{+}[-1], \quad \psi=1_{-}[+1],
$$

and the most general, renormalizable scalar potential for these fields is given by ${ }^{7}$

$$
\begin{aligned}
V_{\text {scal }}= & m_{\chi}^{2}|\chi|^{2}+\left(m_{\phi}^{2}+\kappa_{\chi}|\chi|^{2}+\kappa_{\psi}|\psi|^{2}\right)(\tilde{\phi} \cdot \phi)+m_{\psi}^{2}|\psi|^{2} \\
& +\frac{\lambda_{1}}{4}(\tilde{\phi} \cdot \tilde{\phi})(\phi \cdot \phi)+\frac{\lambda_{2}}{2}(\tilde{\phi} \cdot \tilde{\phi} \cdot \phi \cdot \phi)+\lambda_{3}\left|\chi^{2}\right||\psi|^{2}+\frac{\lambda_{\chi}}{2}|\chi|^{4}+\lambda_{\psi}|\psi|^{4} \\
& +\left[\frac{\kappa_{1}}{2} \psi \psi(\phi \cdot \phi)+\frac{\kappa_{2}}{2} \chi^{*} \chi^{*}(\phi \cdot \phi)+\frac{1}{2} \lambda_{\chi \psi} \psi \psi \chi \chi+\rho \psi(\tilde{\phi} \cdot \phi \cdot \phi)+\text { h.c. }\right],
\end{aligned}
$$

where the $D_{6}$ singlet contractions are explained in appendix A and we take $\kappa_{1}, \kappa_{2}, \lambda_{\chi \psi}$ and $\rho$ to be real. The ground state of this potential is most easily studied in the limit when

$$
\rho \ll 1, \quad \kappa_{2} \ll 1, \quad \lambda_{\chi \psi} \ll 1 .
$$

\footnotetext{
${ }^{7}$ We do not include the SM Higgs, because its backreaction on the flavon potential is negligible as the flavon VEVs are much larger than the electroweak scale. In turn, the flavons will generate a large mass term for the Higgs, which is just the usual hierarchy problem that we do not address here.
} 


\begin{tabular}{|c|cc|cc|}
\hline Observable & $\Delta\left(\mathrm{QL} \nu_{M}-1\right)$ & $\mathrm{Pull}\left(\mathrm{QL} \nu_{M}-1\right)$ & $\Delta\left(\mathrm{QL} \nu_{M}-2\right)$ & $\mathrm{Pull}\left(\mathrm{QL} \nu_{M}-2\right)$ \\
\hline$y_{u}$ & 4 & 0.1 & 4 & -1.3 \\
$y_{c}$ & 2 & -0.1 & 2 & -1.4 \\
$y_{t}$ & 1 & -0.0 & 1 & -0.3 \\
$y_{d}$ & 3.8 & 0.7 & 3.8 & 1.6 \\
$y_{s}$ & 1.6 & -0.1 & 1.5 & -1.4 \\
$y_{b}$ & 0.6 & -0.0 & 0.6 & 0.8 \\
$y_{e}$ & 4 & -0.0 & 4 & -0.0 \\
$y_{\mu}$ & 1.2 & -0.0 & 1.2 & -0.0 \\
$y_{\tau}$ & 0.8 & 0.0 & 0.8 & 0.1 \\
$\theta_{13}^{\text {CKM }}$ & 1.3 & -0.1 & 1.2 & 0.4 \\
$\theta_{12}^{\text {CKM }}$ & 1.8 & -0.0 & 1.7 & -0.1 \\
$\theta_{23}^{\text {CKM }}$ & 4.5 & -0.0 & 4 & -0.1 \\
$\delta_{\mathrm{CP}}$ & 5.1 & -0.1 & 3.9 & 0.0 \\
$\Delta m_{21}^{2}$ & 4.7 & 0.1 & 4.7 & 0.5 \\
$\Delta m_{31}^{2}$ & 3.1 & -0.0 & 3 & -0.2 \\
$\theta_{13}^{\text {PMNS }}$ & 1.4 & -0.1 & 1.4 & -1.4 \\
$\theta_{12}^{\text {PMNS }}$ & 1.1 & 0.2 & 1.1 & 2.3 \\
$\theta_{23}^{\text {PMNS }}$ & 0.8 & 0.4 & 0.8 & 1.6 \\
\hline
\end{tabular}

Table 17. Fine-tuning and pulls for the observables of fit $\mathrm{QL} \nu_{M^{-}}-1$ and fit $\mathrm{QL} \nu_{M^{-}}-2$.

For a suitable range of parameters (see below), one can easily show that the ground state at leading order in $\rho$ and $\kappa_{2}$ is given by

$$
v_{1}^{2}=\frac{\lambda_{\chi} m_{\phi}^{2}-\kappa_{\chi} m_{\chi}^{2}}{\kappa_{\chi}^{2}-\lambda_{2} \lambda_{\chi}}, \quad v_{\chi}^{2}=\frac{\lambda_{2} m_{\chi}^{2}-\kappa_{\chi} m_{\phi}^{2}}{\kappa_{\chi}^{2}-\lambda_{2} \lambda_{\chi}} .
$$

There is a symmetry exchanging $\phi_{1} \leftrightarrow \phi_{2}$ in the potential, which are connected by a $D_{6}$ transformation that we can use to assume the large VEV in the $\phi_{1}$ direction without loss of generality. The VEVs of $v_{2}$ and $v_{\psi}$ only arise at $\mathcal{O}(\kappa)$ and $\mathcal{O}(\kappa \rho)$, respectively:

$$
v_{2}^{2}=\frac{\kappa_{2}^{2} v_{\chi}^{2}}{\left(\lambda_{2}-\lambda_{1}\right)^{2}} \frac{v_{\chi}^{2}}{v_{1}^{2}}, \quad v_{\psi}^{2}=\frac{\kappa_{2}^{2} \rho^{2} v_{1}^{2}}{\left(\lambda_{2}-\lambda_{1}\right)^{2}}\left(\frac{v_{\chi}^{2}\left(\kappa_{\chi}^{2}-\lambda_{2} \lambda_{\chi}\right)}{\tilde{m}^{2}}\right)^{2},
$$

with the shorthand notation

$$
\tilde{m}^{2}=\kappa_{\chi}\left(\kappa_{\chi} m_{\psi}^{2}-\kappa_{\psi} m_{\chi}^{2}-\lambda_{3} m_{\phi}^{2}\right)+\kappa_{\psi} \lambda_{\chi} m_{\phi}^{2}+\lambda_{2}\left(\lambda_{3} m_{\chi}^{2}-\lambda_{\chi} m_{\psi}^{2}\right) .
$$

In order to suppress the VEVs of $\phi_{2}$ and $\psi$ sufficiently, i.e. to ensure the validity of e.g. eq. (2.5), we need roughly $v_{2} / v_{1} \sim v_{2} / v_{\chi} \sim \kappa_{2} \lesssim \varepsilon_{\chi}^{2} \sim 10^{-4}$. Such a small coupling 
is technically natural, since in the limit of $\kappa_{2} \rightarrow 0, \lambda_{\chi \psi} \rightarrow 0$ (or $\rho \rightarrow 0$ ) the Lagrangian acquires a larger symmetry. This can be seen from spelling out the third line of the potential explicitly:

$$
V_{\text {scal }} \supset \kappa_{1} \psi^{2} \phi_{1} \phi_{2}+\kappa_{2} \chi^{*} \chi^{*} \phi_{1} \phi_{2}+\lambda_{\chi \psi} \chi^{2} \psi^{2}+\rho \psi\left(\phi_{1}^{2} \phi_{2}^{*}+\phi_{2}^{2} \phi_{1}^{*}\right)+\text { h.c. }
$$

Indeed, this part only breaks the additional $\mathrm{U}(1)^{3}$ symmetry of the scalar kinetic terms (besides the remaining $\left.\mathrm{U}(1)_{F}\right)$ if $\rho \neq 0$ and $\kappa \neq 0$ or $\lambda_{\chi \psi} \neq 0$.

This observation is also crucial to understand why the additional field $\psi$ is needed: its coupling $\rho$ is the only parameter that breaks the $\mathrm{U}(1)$ symmetry under which $\chi$ is neutral and $\phi_{1}$ and $\phi_{2}$ carry opposite charges. Moreover, it makes clear that we expect (in addition to the massless $\mathrm{U}(1)_{F}$ Goldstone) a very light pseudoscalar in the spectrum whose mass is suppressed by the small couplings $\kappa_{2}, \lambda_{\chi \psi}$ and $\rho$.

After these analytical considerations we finally provide a numerical example, taking the following set of parameters:

$$
\begin{aligned}
& m_{\phi}^{2}=-2 m^{2}, \quad m_{\chi}^{2}=-3 / 10 m^{2}, \quad m_{\psi}^{2}=2 m^{2}, \quad \lambda_{1}=1, \quad \lambda_{2}=1 / 9, \quad \lambda_{\chi}=1, \quad \kappa_{\chi}=-1 / 8 \\
& \lambda_{3}=2 / 3, \quad \lambda_{\chi \psi}=-1 / 20, \quad \kappa_{1}=-1 / 3, \quad \kappa_{\psi}=7 / 10, \quad \rho=-1 / 20, \quad \lambda_{\psi}=9 / 10, \quad \kappa_{2}=1 / 2000 .
\end{aligned}
$$

The absolute minimum in the potential can be calculated numerically, and agrees very well with the above approximate results in eq. (C.4) and eq. (C.5). The VEVs are given by

$$
v_{1}=4.6 m, \quad v_{2}=-3.6 \cdot 10^{-4} m, \quad v_{\chi}=1.7 m, \quad v_{\psi}=-2.1 \cdot 10^{-5} \mathrm{~m},
$$

and therefore

$$
\varepsilon_{\phi}=0.024\left(\frac{190 m}{\Lambda}\right), \quad \varepsilon_{\chi}=0.009\left(\frac{190 m}{\Lambda}\right)
$$

Finally, the scalar mass spectrum is given by one massless Goldstone, 6 massive scalars with masses $\{6.3,6.3,6.0,6.0,3.9,2.6\} \mathrm{m}$ and a light scalar with mass $2.9 \cdot 10^{-5} \mathrm{~m}$. Using the lower bound on $\Lambda$ from section 4.2 (corresponding to an axion mass $m_{a} \lesssim 14 \mathrm{meV}$ ), we get a lower bound on $m$ roughly given by $m \sim \Lambda / 190>5 \cdot 10^{8} \mathrm{GeV}$, so the light scalar has a mass $\gtrsim 15 \mathrm{TeV}$.

We finally comment on the small value of $\kappa_{2}=1 / 2000$ used in the benchmark point. As it is clear from eq. (C.5), small $\kappa_{2}$ ensures the approximate alignment of the doublet VEV along $v_{1}$. This small value is technically natural within the benchmark point, since the renormalization group equation for $\kappa_{2}$ is of the form $d \kappa_{2} / d t \sim \lambda_{\chi \psi}^{*} \kappa_{1} / 16 \pi^{2}$, so radiative corrections to $\kappa_{2}$ are under control. Within the context of a supersymmetric UV completion there might be a more natural possibility to ensure the VEV alignment of $\phi$.

Open Access. This article is distributed under the terms of the Creative Commons Attribution License (CC-BY 4.0), which permits any use, distribution and reproduction in any medium, provided the original author(s) and source are credited. 


\section{References}

[1] F. Feruglio, Pieces of the Flavour Puzzle, Eur. Phys. J. C 75 (2015) 373 [arXiv: 1503.04071] [INSPIRE].

[2] R. Barbieri, G.R. Dvali and L.J. Hall, Predictions from a U(2) flavor symmetry in supersymmetric theories, Phys. Lett. B 377 (1996) 76 [hep-ph/9512388] [INSPIRE].

[3] R. Barbieri, L.J. Hall and A. Romanino, Consequences of a U(2) flavor symmetry, Phys. Lett. B 401 (1997) 47 [hep-ph/9702315] [INSPIRE].

[4] R.G. Roberts, A. Romanino, G.G. Ross and L. Velasco-Sevilla, Precision Test of a Fermion Mass Texture, Nucl. Phys. B 615 (2001) 358 [hep-ph/0104088] [INSPIRE].

[5] R. Dermisek and S. Raby, Fermion masses and neutrino oscillations in $\mathrm{SO}(10)$ SUSY GUT with $D_{3} \times \mathrm{U}(1)$ family symmetry, Phys. Rev. D 62 (2000) 015007 [hep-ph/9911275] [INSPIRE].

[6] E. Dudas, G. von Gersdorff, S. Pokorski and R. Ziegler, Linking Natural Supersymmetry to Flavour Physics, JHEP 01 (2014) 117 [arXiv:1308.1090] [INSPIRE].

[7] A. Falkowski, M. Nardecchia and R. Ziegler, Lepton Flavor Non-Universality in B-meson Decays from a $\mathrm{U}(2)$ Flavor Model, JHEP 11 (2015) 173 [arXiv:1509.01249] [INSPIRE].

[8] LHCb collaboration, Test of lepton universality using $B^{+} \rightarrow K^{+} \ell^{+} \ell^{-}$decays, Phys. Rev. Lett. 113 (2014) 151601 [arXiv: 1406.6482] [INSPIRE].

[9] LHCb collaboration, Test of lepton universality with $B^{0} \rightarrow K^{* 0} \ell^{+} \ell^{-}$decays, JHEP 08 (2017) 055 [arXiv : 1705.05802] [INSPIRE].

[10] F. Wilczek, Axions and Family Symmetry Breaking, Phys. Rev. Lett. 49 (1982) 1549 [INSPIRE].

[11] L. Calibbi, F. Goertz, D. Redigolo, R. Ziegler and J. Zupan, Minimal axion model from flavor, Phys. Rev. D 95 (2017) 095009 [arXiv: 1612.08040] [INSPIRE].

[12] Y. Ema, K. Hamaguchi, T. Moroi and K. Nakayama, Flaxion: a minimal extension to solve puzzles in the standard model, JHEP 01 (2017) 096 [arXiv: 1612.05492] [INSPIRE].

[13] S. Antusch and V. Maurer, Running quark and lepton parameters at various scales, JHEP 11 (2013) 115 [arXiv:1306.6879] [INSPIRE].

[14] I. Esteban, M.C. Gonzalez-Garcia, M. Maltoni, I. Martinez-Soler and T. Schwetz, Updated fit to three neutrino mixing: exploring the accelerator-reactor complementarity, JHEP 01 (2017) 087 [arXiv: 1611.01514] [INSPIRE].

[15] NuFIT 3.2, (2018) www.nu-fit.org.

[16] KATRIN collaboration, A. Osipowicz et al., KATRIN: A Next generation tritium beta decay experiment with sub-eV sensitivity for the electron neutrino mass. Letter of intent, hep-ex/0109033 [INSPIRE].

[17] Planck collaboration, N. Aghanim et al., Planck 2018 results. VI. Cosmological parameters, arXiv: 1807.06209 [INSPIRE].

[18] EUCLID collaboration, R. Laureijs et al., Euclid Definition Study Report, arXiv:1110.3193 [INSPIRE]. 
[19] L. Amendola et al., Cosmology and fundamental physics with the Euclid satellite, Living Rev. Rel. 21 (2018) 2 [arXiv:1606.00180] [INSPIRE].

[20] S. Dell'Oro, S. Marcocci, M. Viel and F. Vissani, Neutrinoless Double Beta Decay: 2015 Review, Adv. High Energy Phys. 2016 (2016) 2162659 [arXiv: 1601.07512] [InSPIRE].

[21] M. Dine, W. Fischler and M. Srednicki, A Simple Solution to the Strong CP Problem with a Harmless Axion, Phys. Lett. B 104 (1981) 199 [INSPIRE].

[22] A.R. Zhitnitsky, On Possible Suppression of the Axion Hadron Interactions (in Russian), Sov. J. Nucl. Phys. 31 (1980) 260 [INSPIRE].

[23] J.E. Kim, Weak Interaction Singlet and Strong CP Invariance, Phys. Rev. Lett. 43 (1979) 103 [INSPIRE].

[24] M.A. Shifman, A.I. Vainshtein and V.I. Zakharov, Can Confinement Ensure Natural CP Invariance of Strong Interactions?, Nucl. Phys. B 166 (1980) 493 [INSPIRE].

[25] L. Di Luzio, F. Mescia, E. Nardi, P. Panci and R. Ziegler, Astrophobic Axions, Phys. Rev. Lett. 120 (2018) 261803 [arXiv: 1712.04940] [INSPIRE].

[26] G. Grilli di Cortona, E. Hardy, J. Pardo Vega and G. Villadoro, The QCD axion, precisely, JHEP 01 (2016) 034 [arXiv: 1511.02867] [INSPIRE].

[27] S. Borsányi et al., Calculation of the axion mass based on high-temperature lattice quantum chromodynamics, Nature 539 (2016) 69 [arXiv:1606.07494] [INSPIRE].

[28] CLEO collaboration, R. Ammar et al., Search for the familon via $B^{ \pm} \rightarrow \pi^{ \pm} X^{0}$, $B^{ \pm} \rightarrow K^{ \pm} X^{0}$ and $B^{ \pm} \rightarrow K_{S}^{0} X^{0}$ decays, Phys. Rev. Lett. 87 (2001) 271801 [hep-ex/0106038] [INSPIRE].

[29] E787 and E949 collaborations, S. Adler et al., Measurement of the $K^{+} \rightarrow \pi^{+} \nu \bar{\nu}$ branching ratio, Phys. Rev. D 77 (2008) 052003 [arXiv:0709.1000] [InSPIRE].

[30] A. Jodidio et al., Search for Right-Handed Currents in Muon Decay, Phys. Rev. D 34 (1986) 1967 [Erratum ibid. D 37 (1988) 237] [INSPIRE].

[31] R.D. Bolton et al., Search for Rare Muon Decays with the Crystal Box Detector, Phys. Rev. D 38 (1988) 2077 [INSPIRE].

[32] J.T. Goldman et al., Light Boson Emission in the Decay of the $\mu^{+}$, Phys. Rev. D 36 (1987) 1543 [INSPIRE].

[33] M.M. Miller Bertolami, B.E. Melendez, L.G. Althaus and J. Isern, Revisiting the axion bounds from the Galactic white dwarf luminosity function, JCAP 10 (2014) 069 [arXiv: 1406.7712] [INSPIRE].

[34] W. Keil, H.-T. Janka, D.N. Schramm, G. Sigl, M.S. Turner and J.R. Ellis, A Fresh look at axions and SN-1987A, Phys. Rev. D 56 (1997) 2419 [astro-ph/9612222] [INSPIRE].

[35] ARGUS collaboration, H. Albrecht et al., A Search for lepton flavor violating decays $\tau \rightarrow e \alpha, \tau \rightarrow \mu \alpha, Z$. Phys. C 68 (1995) 25 [INSPIRE].

[36] J.H. Chang, R. Essig and S.D. McDermott, Supernova 1987A Constraints on Sub-GeV Dark Sectors, Millicharged Particles, the QCD Axion and an Axion-like Particle, arXiv: 1803.00993 [INSPIRE]. 
[37] G. Anelli et al., Proposal to Measure the Rare Decay $K^{+} \rightarrow \pi^{+} \nu \bar{\nu}$ at the CERN SPS, CERN-SPSC-2005-013 (2005) [SPSC-P-326] [INSPIRE].

[38] NA62 collaboration, R. Fantechi, The NA62 experiment at CERN: status and perspectives, in proceedings of the 12th Conference on Flavor Physics and CP Violation (FPCP 2014), Marseille, France, 26-30 May 2014, arXiv:1407.8213, http://inspirehep.net/record/1309159/files/arXiv:1407.8213.pdf [INSPIRE].

[39] J. Preskill, M.B. Wise and F. Wilczek, Cosmology of the Invisible Axion, Phys. Lett. B 120 (1983) 127 [INSPIRE].

[40] L.F. Abbott and P. Sikivie, A Cosmological Bound on the Invisible Axion, Phys. Lett. B 120 (1983) 133 [INSPIRE].

[41] M. Dine and W. Fischler, The Not So Harmless Axion, Phys. Lett. B 120 (1983) 137 [INSPIRE].

[42] Particle Data Group collaboration, C. Patrignani et al., Review of Particle Physics, Chin. Phys. C 40 (2016) 100001 [INSPIRE].

[43] ADMX collaboration, S.J. Asztalos et al., An Improved RF cavity search for halo axions, Phys. Rev. D 69 (2004) 011101 [astro-ph/0310042] [INSPIRE].

[44] L. Di Luzio, F. Mescia and E. Nardi, Redefining the Axion Window, Phys. Rev. Lett. 118 (2017) 031801 [arXiv: 1610.07593] [INSPIRE].

[45] S. Hannestad, A. Mirizzi, G.G. Raffelt and Y.Y.Y. Wong, Neutrino and axion hot dark matter bounds after WMAP-7, JCAP 08 (2010) 001 [arXiv: 1004.0695] [INSPIRE].

[46] M. Archidiacono, S. Hannestad, A. Mirizzi, G. Raffelt and Y.Y.Y. Wong, Axion hot dark matter bounds after Planck, JCAP 10 (2013) 020 [arXiv:1307.0615] [INSPIRE].

[47] E. Di Valentino, E. Giusarma, M. Lattanzi, O. Mena, A. Melchiorri and J. Silk, Cosmological Axion and neutrino mass constraints from Planck 2015 temperature and polarization data, Phys. Lett. B 752 (2016) 182 [arXiv:1507.08665] [INSPIRE].

[48] A. Ayala, I. Domínguez, M. Giannotti, A. Mirizzi and O. Straniero, Revisiting the bound on axion-photon coupling from Globular Clusters, Phys. Rev. Lett. 113 (2014) 191302 [arXiv: 1406.6053] [INSPIRE].

[49] R. Bähre et al., Any light particle search II —Technical Design Report, 2013 JINST 8 T09001 [arXiv: 1302.5647] [INSPIRE].

[50] CAST collaboration, V. Anastassopoulos et al., New CAST Limit on the Axion-Photon Interaction, Nature Phys. 13 (2017) 584 [arXiv:1705.02290] [INSPIRE].

[51] IAXO collaboration, I. Irastorza et al., The International Axion Observatory IAXO. Letter of Intent to the CERN SPS committee, CERN-SPSC-2013-022 [INSPIRE].

[52] E. Armengaud et al., Conceptual Design of the International Axion Observatory (IAXO), 2014 JINST 9 T05002 [arXiv:1401.3233] [INSPIRE].

[53] ADMX collaboration, S.J. Asztalos et al., A SQUID-based microwave cavity search for dark-matter axions, Phys. Rev. Lett. 104 (2010) 041301 [arXiv:0910.5914] [INSPIRE].

[54] ADMX collaboration, C. Hagmann et al., Results from a high sensitivity search for cosmic axions, Phys. Rev. Lett. 80 (1998) 2043 [astro-ph/9801286] [INSPIRE]. 
[55] MADMAX Working Group, A. Caldwell et al., Dielectric Haloscopes: A New Way to Detect Axion Dark Matter, Phys. Rev. Lett. 118 (2017) 091801 [arXiv: 1611.05865] [INSPIRE].

[56] C.D. Froggatt and H.B. Nielsen, Hierarchy of Quark Masses, Cabibbo Angles and CP-violation, Nucl. Phys. B 147 (1979) 277 [INSPIRE].

[57] A. Ernst, A. Ringwald and C. Tamarit, Axion Predictions in $\mathrm{SO}(10) \times \mathrm{U}(1)_{\mathrm{PQ}}$ Models, JHEP 02 (2018) 103 [arXiv: 1801.04906] [INSPIRE].

[58] A. Blum, C. Hagedorn and M. Lindner, Fermion Masses and Mixings from Dihedral Flavor Symmetries with Preserved Subgroups, Phys. Rev. D 77 (2008) 076004 [arXiv:0709.3450] [INSPIRE].

[59] W. Grimus and P.O. Ludl, Finite flavour groups of fermions, J. Phys. A 45 (2012) 233001 [arXiv:1110.6376] [INSPIRE]. 